\title{
Apports et limites de la série comptable à la connaissance de la châtellenie de Saffré au $\mathrm{XV}^{\mathrm{e}}$ siècle
}

The contributions and limitations of the accounts of the châtellenie of Saffré in the fifteenth century

\section{Brice Rabot}

\section{OpenEdition}

\section{Journals}

Édition électronique

URL : https://journals.openedition.org/abpo/4580

DOI : $10.4000 / a b p o .4580$

ISBN : 978-2-7535-7968-2

ISSN : 2108-6443

Éditeur

Presses universitaires de Rennes

\section{Édition imprimée}

Date de publication : 20 novembre 2019

Pagination : 21-48

ISBN : 978-2-7535-7966-8

ISSN : 0399-0826

Référence électronique

Brice Rabot, « Apports et limites de la série comptable à la connaissance de la châtellenie de Saffré au $x v^{e}$ siècle », Annales de Bretagne et des Pays de l'Ouest [En ligne], 126-3 | 2019, mis en ligne le 01 janvier 2022, consulté le 08 janvier 2022. URL : http://journals.openedition.org/abpo/4580 ; DOI : https:// doi.org/10.4000/abpo.4580 


\title{
Apports et limites de la série comptable à la connaissance de la châtellenie de Saffré au Xve siècle
}

\author{
Brice RABOT \\ Docteur en histoire, chercheur associé au CRHIA, université de Nantes, et au CESCM, \\ université de Poitiers
}

Au carrefour des campagnes intérieures et des marches orientales du comté de Nantes, la châtellenie de Saffré occupe une position stratégique à la fin du Moyen Âge. Les échanges commerciaux, les incursions militaires dues aux grandes opérations de la seconde moitié du $\mathrm{XV}^{\mathrm{e}}$ siècle ou encore la proximité des voies de communication comme l'Isac, vers la Vilaine, et l'Erdre, vers Nantes et la Loire, contribuent à organiser le territoire et à faire prospérer cette châtellenie.

La châtellenie de Saffré n'a toutefois pas jusqu'à présent retenu l'attention des historiens. Contrairement aux autres seigneuries des marches orientales - comme Belligné ${ }^{1}$, Varades ${ }^{2}$ ou Ancenis ${ }^{3}$ - ou celles des marches méridionales - comme les Huguetières ${ }^{4}$-, la châtellenie de Saffré n'a pas fait l'objet de mémoire universitaire alors même que des comptes existent pour cette seigneurie. Ces séries comptables ont en effet servi de point de départ à cette étude grâce à la diversité des informations recueillies, mais aussi et surtout grâce à la continuité chronologique due à la conservation croissante des registres dans la seconde moitié du Xve siècle. Les comptes de la châtellenie de Saffré sont rassemblés dans la sous-série 1 E des Archives départementales de Loire-Atlantique, à l'inverse de ceux des autres châtel-

1. Termeau, Dominique, La châtellenie de Belligné de la fin de l'époque médiévale aux débuts des Temps modernes (1444-1531), mémoire de maîtrise (dactyl.), Université de Nantes, 1980.

2. Reverseau, Vincent, La châtellenie de Varades à la fin du Moyen Âge et au début de l'époque moderne (1466-1523), mémoire de maîtrise (dactyl.), Université de Nantes, 1996.

3. Vigneau, Thomas, Les métairies de la prévôté d'Ancenis à la fin du Moyen Âge (vers 1450-1525), mémoire de maîtrise (dactyl.), Université de Nantes, 1994.

4. SADDER, Évelyne, Une seigneurie rurale du pays nantais au XVe siècle : les Huguetières à travers les comptes du receveur, mémoire de des (dactyl.), Centre universitaire de Savoie, Chambéry, 1975. 
lenies, qui ont été versés dans la série E consacrée au "Trésor des chartes " des ducs de Bretagne. La sous-série 1 E est aujourd'hui encore difficile d'accès pour les chercheurs : ses fonds n'ont fait l'objet que d'inventaires très sommaires, qui obligent à recourir à des sondages multiples et systématiques pour saisir les apports et les limites de ces sources. De même, les aveux ou les dénombrements de rentes et de droits pour la châtellenie de Saffré sont quasi-inexistants, contrairement aux autres châtellenies. Les comptes s'avèrent, dans ces conditions, d'autant plus précieux qu'ils sont les seules sources disponibles pour étudier la seconde moitié du Xve siècle. Les croisements avec les aveux et les minus ${ }^{5}$ sont également moins aisés que pour les autres châtellenies : la baronnie de Châteaubriant, implantée autour de la ville éponyme, est à $32 \mathrm{~km}$, tandis que le domaine ducal du Gâvre est distant de 14 km de Saffré. Tous ces éléments expliquent sans doute que les historiens aient jusqu'ici délaissé la châtellenie de Saffré.

Il est pourtant fort intéressant d'étudier la comptabilité de cette châtellenie. Comme dans les autres châtellenies évoquées plus haut, la série de comptes de Saffré permet d'éclairer différemment des événements bien connus et étudiés depuis les années 1980-1990 : quels sont les impacts territoriaux et structurels des grandes crises des années 1460-1480? Comment les " châtelains receveurs ${ }^{6}$ " y font-ils face? Quels outils utilisent-ils pour maintenir les revenus seigneuriaux dans une période d'incertitude croissante? Ces questions font aujourd'hui l'objet de relectures en jouant sur les angles d'approche et les échelles d'analyse, du niveau local à celui du sous-ensemble régional.

Cet article se propose donc de revenir sur ces problématiques en prenant appui sur les comptabilités du $\mathrm{Xv}^{\mathrm{e}}$ siècle. L'objectif premier sera de mettre en évidence les principaux rouages de la châtellenie de Saffré au long du Xve siècle, pour mieux comprendre l'organisation du territoire. Nous chercherons également à dégager les signes d'adaptation pour voir si la châtellenie de Saffré s'inscrit dans les lignes observées pour les autres structures seigneuriales ou si, au contraire, elle tend à s'en écarter. Cette réflexion nous conduira à analyser les indices laissés par les " châtelains receveurs " dans leur comptabilité. Après avoir présenté la châtellenie et sa série comptable, nous nous focaliserons sur les structures du prélèvement, pour terminer par l'étude des outils de gestion.

5. Les minus décrivent, par " le menu ", les rentes, revenus et terres possédés par les seigneurs au moment du rachat, suite au décès du détenteur précédent. Ils n'oublient pas de relever les droits détenus sur les hommes et les possessions foncières. Pour une présentation plus détaillée des minus et de leurs apports à la connaissance historique de la Bretagne méridionale, voir : RABOT, Brice, Les structures seigneuriales rurales en Bretagne méridionale (XIV-XVI siècles), Rennes, PUR, 2017, p. 38-44.

6. Nom donné, dans les registres de compte, aux agents seigneuriaux chargés de mettre en valeur la châtellenie de Saffré et de percevoir tous les droits dus. Les " châtelains receveurs " sont amenés, dans le cadre de leurs fonctions, à présenter au terme de leur charge les cahiers de compte. Ces registres sont ensuite examinés par des proches des seigneurs, que les comptes appellent " auditeurs". 


\section{Remarques préliminaires sur la série comptable}

\section{Présentation matérielle des comptes}

Les comptes de la châtellenie de Saffré ${ }^{7}$ ne sont pas différents de ceux des autres châtellenies de Bretagne méridionale. Rédigés sur papier, ils sont organisés autour de la même trame que les autres séries, elles-mêmes calquées sur le modèle développé par la Chambre des comptes de Bretagne à la fin du Moyen Âge ${ }^{8}$. Divisés en deux grandes parties, les " charges et recettes " suivies des "mises et dépenses ", les comptes sont globalement bien tenus. Le faible nombre de ratures en atteste ${ }^{9}$. Les grandes rubriques sont subdivisées en sections qui reprennent l'ordre établi par la Chambre des comptes : les "rentes certaines" en deniers, autrement dit les rentes et les droits pesant sur les biens concédés comme les censives, constituent sans surprise l'essentiel des recettes, complétées par les "rentes incertaines " en deniers, qui proviennent des fermes et des mises aux enchères ${ }^{10}$. Sont ensuite présentées les rentes en nature, elles-mêmes réparties en " rentes certaines " et " incertaines ". Les secondes sont, là encore, beaucoup plus limitées ${ }^{11}$.

Les cahiers sont reliés les uns aux autres par des ficelles fines, témoins de la volonté de conserver les registres dans de bonnes conditions. Les renvois aux registres précédents à l'intérieur des folios, la lisibilité générale des comptes et l'ornementation reflètent, comme pour les autres châtellenies, l'importance accordée aux registres. Preuves des droits seigneuriaux mais aussi réceptacles des revenus perçus, les comptes sont empreints d'une charge symbolique à laquelle les chercheurs sont de plus en plus sensibles ${ }^{12}$.

La série comptable de Saffré forme un ensemble tout à fait cohérent sur le plan chronologique, de 1446 à 1481. En dépit des lacunes (tableau 1), liées aux aléas de la conservation, il est possible de retracer les grandes perspectives avant et après les soubresauts des années 1460-1470, ou encore

7. Les neuf registres de comptes de la châtellenie de Saffré pour le $\mathrm{XV}^{\mathrm{e}}$ siècle sont aujourd'hui classés sous la même cote : Arch. dép. de Loire-Atlantique, 1 E 674.

8. Les premiers comptes connus en Bretagne remontent au XIV siècle (KERHERVÉ, Jean, L'État breton aux XIV et XV $V^{e}$ siècles. Les ducs, l'argent et les hommes, Paris, Maloine, 1987, t. 1, p. 411-416). Des comptes et des déductions sont aussi signalés sous le règne de Jean II (1286-1305), à la fin du XIII ${ }^{\mathrm{e}}$ siècle, mais aucun ne nous est parvenu (ibidem, p. 145).

9. Les deux premiers registres de la série ne comptent par exemple aucune rature pour les sommes ou les rentes (Arch. dép. de Loire-Atlantique, 1 E 674 (1) et 1 E 674 (2).

10. Comme dans les autres seigneuries bretonnes, les mises aux enchères s'effectuent à la chandelle. Ce mode d'attribution permet de tirer un profit supérieur grâce à la mise en concurrence des preneurs.

11. Nous reviendrons sur cette question du prélèvement dans la seconde partie de ce texte.

12. RABOt, Brice, "Progrès et diffusion des techniques comptables à la fin du Moyen Âge : l'exemple de la Bretagne méridionale ", Comptabilité(s), nº 10, 2018. [https://journals.openedition.org/comptabilites/3119]. 
avant la grande crise des années 1480-149013. L'absence de registres conservés pour cette dernière période rend impossible l'examen des impacts des affrontements avec la France. La crise de 1468, en revanche, est connue grâce au registre de $1474^{14}$, qui fait clairement référence aux conséquences de l'incursion française dans les marches d'Ancenis à l'automne 1468.

\section{Tableau 1 - Années couvertes par les registres de compte}

\begin{tabular}{|c|c|c|}
\hline Cote & Lieu de conservation & Données disponibles $^{\mathrm{a}}$ \\
\hline $1 \mathrm{E} 674(1)$ & \multirow{9}{*}{ Arch. dép. de Loire-Atlantique } & 1446 \\
\hline $1 \mathrm{E} 674(2)$ & & 1447 \\
\hline $1 \mathrm{E} 674$ (3) & & 1454 \\
\hline $1 \mathrm{E} 674$ (4) & & 1458 \\
\hline $1 \mathrm{E} 674(5)$ & & 1462 et 1463 \\
\hline $1 \mathrm{E} 674$ (6) & & $1462,1463,1464$ et 1465 \\
\hline $1 \mathrm{E} 674(7)$ & & $1470,1471,1472$ et 1473 \\
\hline $1 \mathrm{E} 674$ (8) & & 1475 \\
\hline $1 \mathrm{E} 674(9)$ & & 1480 \\
\hline
\end{tabular}

a. Ne sont présentées ici que les années complètes. Pour les dates de début et de conclusion des registres, nous renvoyons au tableau 2 , infra.

\section{Les receveurs}

Comme les autres séries comptables évoquées ci-dessus, celle de Saffré fournit quelques précisions sur les " châtelains receveurs". Ils sont chargés de percevoir les revenus et les droits pour leur seigneur, les sires et dames de la Hunaudaye, qu'ils présentent toujours en introduction de leur registre. Ils sont quasiment les seuls officiers seigneuriaux à avoir transmis leur nom, les autres n'étant le plus souvent pas directement nommés dans les folios, en dehors des " mises et dépenses ». Les destinataires des «mises et paiements " sont en revanche clairement désignés pour faciliter les opérations de contrôle à la fin des exercices comptables, en lien avec les opérations d'audition ${ }^{15}$. Les informations contenues dans les registres peuvent être rassemblées dans le tableau suivant (tableau 2).

13. Plus connue sous le nom de "guerre d'indépendance de Bretagne " (1487-1491), cette crise marque en profondeur les structures seigneuriales et économiques, fragilisant les campagnes pour plusieurs décennies. Les premiers effets de la reconstruction agraire ne se font pas sentir avant le milieu du siècle suivant (LEGUAY, Jean-Pierre, MARTIN, Hervé, Fastes et malheurs de la Bretagne ducale (1213-1532), Rennes, Éditions OuestFrance Université, 1997, p. 194-209).

14. Arch. dép. de Loire-Atlantique, 1 E 674 (7).

15. Nous apprenons, en lisant attentivement cette rubrique, que les comptes sont en fait tenus par Guillaume de Kerduel et Pierre Rembaut : " [...] Item, a poié à la despanse des chevaulx de Kerduel et de Pierre Rembaut qui tenoient les comptes 1 septier [d'avoine]" 
Tableau 2 - Durée des charges et noms des "châtelains receveurs "

\begin{tabular}{|c|c|c|c|c|c|}
\hline Cote & $\begin{array}{c}\text { Durée de } \\
\text { l'exercice } \\
\text { comptable }\end{array}$ & $\begin{array}{l}\text { Nom et } \\
\text { prénom }\end{array}$ & $\begin{array}{c}\text { Début de la } \\
\text { charge }\end{array}$ & $\begin{array}{l}\text { Fin de la } \\
\text { charge }\end{array}$ & $\begin{array}{c}\text { Longueur } \\
\text { du registre } \\
\text { (nombre de } \\
\text { folios) }\end{array}$ \\
\hline $1 \mathrm{E} 674$ (1) & 1 an & \multirow{6}{*}{ Yvon Deraye } & 14 juin 1446 & 9 juillet 1447 & 15 \\
\hline 1 E 674 (2) & 1 an & & 9 juillet 1447 & $1^{\mathrm{er}}$ août 1448 & 16 \\
\hline 1 E 674 (3) & 1 an & & $\begin{array}{c}22 \text { janvier } \\
1455 \text { (n. st.) }\end{array}$ & $\begin{array}{c}15 \text { janvier } \\
1456 \text { (n. st.) }\end{array}$ & 21 \\
\hline 1 E 674 (4) & 1 an & & 18 mai 1458 & 18 avril 1459 & 11 \\
\hline 1 E 674 (5) & 2 ans & & $1462^{a}$ & 16 mai 1464 & 24 \\
\hline 1 E 674 (6) & 4 ans & & $\begin{array}{c}21 \text { mars } \\
1461\end{array}$ & 27 juin 1466 & 42 \\
\hline 1 E $674(7)$ & 5 ans & \multirow{3}{*}{$\begin{array}{c}\text { Jehan Pae- } \\
\text { tral }\end{array}$} & $\begin{array}{c}18 \text { mars } \\
1470 \text { (n. st.) }\end{array}$ & $\begin{array}{c}26 \text { mars } \\
1474 \text { (n. st.) }\end{array}$ & 62 \\
\hline 1 E 674 (8) & 1 an & & 21 juin 1475 & 13 mai 1476 & 18 \\
\hline 1 E 674 (9) & 1 an & & 21 juin 1480 & $\begin{array}{c}24 \text { juillet } \\
1481\end{array}$ & 39 \\
\hline
\end{tabular}

a. La perte du folio d'introduction empêche aujourd'hui de connaître la date exacte d'entrée en charge du receveur. Le registre débute en 1462, car les rentes de cette annéelà sont retranscrites. Le nom du prédécesseur d'Yvon Deraye est en revanche connu : Jehan de L'Espinay, " precedent châtelain ", sans que l'on connaisse ses dates d'exercice (Arch. dép. de Loire-Atlantique, 1 E 674 (5), fo 6 ro).

Une première chose frappe à l'examen du tableau. Contrairement aux autres châtellenies, où plusieurs receveurs sont recensés, deux seulement apparaissent distinctement dans les registres de la seconde moitié du $\mathrm{XV}^{\mathrm{e}}$ siècle ${ }^{16}$. Une autre originalité doit être soulignée : ici, chaque receveur se maintient d'un exercice à l'autre, sur plusieurs décennies, alors que les autres séries comptables attestent plutôt d'une alternance, qui permet aux receveurs de retrouver leur charge quelques années après leur(s) successeur(s). Comment expliquer cette situation? Yvon Deraye et Jehan

(Arch. dép. de Loire-Atlantique, 1 E 674 (5), fo $38 \mathrm{r}^{\circ}$ ). Ces hommes apparaissent de nouveau aux côtés des auditeurs, c'est-à-dire des hommes proches de l'entourage seigneurial chargés de vérifier l'exactitude des informations présentées par les receveurs dans les registres avant de clore les exercices comptables. L'audition des comptes donnait lieu à une procédure codifiée et très stricte, sur le modèle établi et diffusé par la Chambre des comptes ducale à la fin du XIV ${ }^{\mathrm{e}}$ siècle. Les auditeurs contrôlaient scrupuleusement les informations des receveurs en ajoutant, le cas échéant, des remarques dans les marges latérales des registres pour valider ou, au contraire, rejeter les demandes de rabais par exemple. Sur ce sujet, voir : KERHERVÉ, Jean, L'État breton..., op. cit., t. 1, p. 363; RABOT, Brice, "Progrès et diffusion des techniques comptables... ", art. cité.

16. Auquel il faut adjoindre Jehan de L'Espinay, portant à trois le nombre de « châtelains receveurs " exerçant à Saffré entre 1446 et 1480. 
Paetral sont, nous devons le reconnaître, en grande partie inconnus. Aucune information précise ne transparaît quant à leur patrimoine, leur origine ou encore leurs liens avec le monde seigneurial. Les aléas de la conservation compliquent la tâche du chercheur. Les cinquième et sixième registres de la série comptable de Saffré se recoupent en partie. Le cinquième concerne le relevé des fermes, des "ventes et lots ", les charges en nature (en céréales [froment, seigle et avoine] et en volailles). Il se termine par la présentation des mises et des dépenses effectuées entre avril 1463 et mars 1464. Yvon Deraye choisit donc de reprendre, dans le sixième registre ${ }^{17}$, l'ensemble des données disponibles entre mars 1461 et juin 1466 pour présenter un bilan suffisamment clair et documenté. Le cinquième registre a très certainement été conservé dans les fonds pour servir de preuve. Le registre de référence pour les années 1462 et 1463 n'est par conséquent pas le cinquième, mais bien le sixième.

Leur reconduction sur plusieurs décennies démontre la valeur et les aptitudes professionnelles des "châtelains receveurs". Qualifiés ${ }^{18}$, parfaits connaisseurs des individus et du terrain qu'ils étaient amenés à administrer, Yvon Deraye et Jehan Paetral prennent en charge des terres qu'ils concèdent à d'autres tenanciers ${ }^{19}$. Ils se rapprochent donc des paysans qu'ils côtoient régulièrement au moment du versement des redevances. Les gages des receveurs de Saffré restent stables tout au long de la période, car fixés par les usages. Les registres n'apportent toutefois aucun élément sur l'établissement des montants. Les gages sont modérés si on les compare à ceux connus pour les autres châtellenies. Les receveurs des Huguetières perçoivent ainsi des revenus deux fois et demi supérieurs ${ }^{20}$. Comme leurs homologues, Yvon Deraye ${ }^{21}$ et Jehan Paetral disposent par conséquent d'autres sources de revenus, à commencer par l'exploitation de domaines ${ }^{22}$

17. Qui est aussi le dernier qu'il présente en tant que " châtelain receveur " de Saffré.

18. Les " châtelains receveurs " de Saffré sont capables d'additionner les rentes car ils effectuent régulièrement des bilans partiels, qu'ils insèrent dans les registres, à destination des auditeurs (pour faciliter les opérations de vérification et de contrôle des calculs). Ils sont aussi capables de représenter et de défendre les intérêts seigneuriaux dans le cadre des plaids. Ils sont enfin amenés à recueillir régulièrement les informations nécessaires à la mise à jour des listes de rentes : recensement des tenanciers, arpentage du territoire et délimitation des parcelles, etc.

19. Yvon Bouinière, " châtelain receveur " des Huguetières, présente le même profil avec l'exploitation de terres concédées en sus de sa charge (SADDIER, Évelyne, Une seigneurie rurale..., op. cit., p. 32 ).

20. Tout au long du $\mathrm{Xv}^{\mathrm{e}}$ siècle, les gages des receveurs des Huguetières s'élèvent à 20 livres annuelles (SADDIER, Évelyne, Une seigneurie rurale..., op. cit., p. 28).

21. Le compte rendu en 1476 l'indique clairement : " [...] Et de la ferme de la maison o son jardrin qui aultrefoiz fut à Yvon Deraye cedit receveur pour le temps de cedit compte ne s'en charge de nulle recepte pour ce que n'a trouvé à qui l'affermer " (Arch. dép. de Loire-Atlantique, 1 E 674 (8), fo $3 \mathrm{r}^{\circ}$ ).

22. Dans le compte rendu en 1481, Jehan Paetral est cité parmi les tenanciers pour les nouvelles baillées, avec une redevance annuelle de 5 sous due chaque année à l'Angevine (Notre-Dame angevine : 8 septembre) pour une tenure sise à Saffré (Arch. dép. de LoireAtlantique, 1 E 674 (9), fo $5 \mathrm{v}^{\mathrm{o}}$ ). 
tenus " de leur seigneur ${ }^{23}$ ", pour subvenir à leurs besoins et leur procurer des ressources annuelles suffisantes. Nous ne connaissons malheureusement pas le produit réel de ces domaines ${ }^{24}$. Les sénéchaux, chargés de mettre en œuvre la justice seigneuriale avec les plaids, perçoivent eux aussi six livres de revenus annuels (tableau 3). Les " châtelains receveurs " se hissent donc, par leurs gages, au niveau des officiers seigneuriaux supérieurs. Ils occupent par conséquent une place centrale dans les structures de commandement, ce qui justifie qu'ils touchent les mêmes gages que les grands officiers judiciaires (les sénéchaux). Les sires de la Hunaudaye ont parfaitement conscience de la modestie des gages alloués aux receveurs; ils n'hésitent pas à leur accorder des sommes ou des avantages supplémentaires pour les remercier et les attacher à leur service :

" [...] Item, audit recepveurs [ sic] pour ses gaiges desdiz ans mil IIIIC ${ }^{\mathrm{C} X I}$ $\{$ LX soulz et des autres\}, LXII, LXIII, LXIIII (LXV) \{jucques en octobre LXV \} lui dit les acroestre et augmenter et en demande poesment et rabat au plesir de monseigneur et de messeigneurs des comptes. \{Pour l'an LXI LX soulz et pour IIII ans commenczés le derrain jour de octobre LXI et finissans en octobre LXV pour chacun an VI livres en est somme XXVII livres ${ }^{25}$."

Les receveurs sont, en définitive, suffisamment proches des seigneurs pour nouer avec eux des relations de confiance. L'examen des conclusions des registres des années 1460 laisse voir la signature du receveur aux côtés de celle des auditeurs ${ }^{26}$, dont la plupart appartiennent à l'entourage proche des seigneurs comme l'indiquent clairement les annotations ${ }^{27}$. Les receveurs entretiennent par ailleurs des liaisons étroites avec les tenanciers. Véritables pivots de la châtellenie, les receveurs font figure d'intendants entre le monde seigneurial et le monde paysan. Ils parcourent à ce titre régulièrement le territoire pour accomplir leurs multiples tâches : effectuer les nouvelles baillées, percevoir les rentes foncières, etc. En se portant acquéreurs de " nouvelles baillées ${ }^{28}$ ", les " châtelains receveurs " se rapprochent en outre du monde paysan. Aucun autre Deraye ou Paetral n'est

23. « [...] Cedit receveur a esplecte et fait ensemencer une piece de terre qui est situe en la boce et gaingnerie du Veil molin de Saffre, qui aultrefoiz fut audit Yvon Deraye, et laquelle fut baillée a mondit seigneur par presaige, au pris de X soulz de rante, cedit receveur s'en poie audit pris (entendu qu'il le) pour toutes charges pour lesdiz termes de l'Engevine et de Noël l'an mil IIII ${ }^{\mathrm{C}}$ LXXV et pour ce X soulz " (Arch. dép. de LoireAtlantique, 1 E 674 (8), $\left.\mathrm{f}^{\circ} 3 \mathrm{v}^{\mathrm{o}}\right)$.

24. Arch. dép. de Loire-Atlantique, B 1874.

25. Arch. dép. de Loire-Atlantique, 1 E 674 (6), fo 30 vo.

26. Le terme " auditeur " désigne dans les comptes seigneuriaux de la fin du Moyen Âge les hommes chargés de vérifier l'exactitude des informations relevées par les receveurs et de valider les exercices comptables.

27. Le seigneur de La Guerche est ainsi mentionné dans le registre rendu en 1466 (Arch. dép. de Loire-Atlantique, 1 E 674 (6), fo $20 \mathrm{r}^{\circ}$ ). Les sires de la Hunaudaye entretiennent des liens féodo-vassaliques avec le châtelain de Fresnay (Plessé) à travers le versement de reconnaissances de fiefs en numéraire ( $c f$. infra).

28. Par « nouvelles baillées " il faut entendre les censives nouvellement mises en culture ou les censives restaurées après la guerre de Succession (1341-1364). 
Brice RABOT

Tableau 3 - Gages des officiers seigneuriaux

\begin{tabular}{|c|c|c|c|c|c|}
\hline Cote & $\begin{array}{l}\text { Référence } \\
\text { dans le } \\
\text { compte }\end{array}$ & Année & Nom de l'officier & $\begin{array}{l}\text { Nature de } \\
\text { l'office }\end{array}$ & \begin{tabular}{|l} 
Montant \\
annuel des \\
gages
\end{tabular} \\
\hline \multirow{3}{*}{1 E 674 (1) } & Fo $^{\circ} 12 \mathrm{v}^{\mathrm{o}}$ & \multirow{3}{*}{1446} & Olivier Germand & Sénéchal & 6 livres \\
\hline & $\mathrm{F}^{\mathrm{o}} 12 \mathrm{v}^{\mathrm{o}}$ & & Jehan Chollet & $\begin{array}{l}\text { Alloué a (cour } \\
\text { de Saffré) }\end{array}$ & 3 livres \\
\hline & $\mathrm{F}^{\mathrm{o}} 13 \mathrm{r}^{\mathrm{o}}$ & & Yvon Deraye & Receveur & 6 livres \\
\hline \multirow{3}{*}{$1 \mathrm{E} 674$ (2) } & \multirow{3}{*}{$\mathrm{F}^{\mathrm{o}} 12 \mathrm{v}^{\mathrm{o}}$} & \multirow{3}{*}{1447} & Olivier Germand & Sénéchal & 6 livres \\
\hline & & & Jehan Chollet & Alloué & 3 livres \\
\hline & & & Yvon Deraye & Receveur & 6 livres \\
\hline \multirow{4}{*}{1 E 674 (3) } & \multirow{4}{*}{$F^{o} 17 r^{o}$} & \multirow{4}{*}{1455} & Olivier Germand & Sénéchal & 6 livres \\
\hline & & & Jehan Chollet & Alloué & 3 livres \\
\hline & & & Jehan Saffré & $\begin{array}{l}\text { Procureur } \\
\text { (cour de } \\
\text { Saffré) }\end{array}$ & 15 sous \\
\hline & & & Yvon Deraye & Receveur & 6 livres \\
\hline \multirow{4}{*}{$1 \mathrm{E} 674(4)$} & $\mathrm{F}^{\circ} 8 \mathrm{r}^{\circ}$ & \multirow{4}{*}{1458} & Pierres Lebotellier & Sénéchal & 6 livres \\
\hline & \multirow{3}{*}{$\mathrm{F}^{\mathrm{o}} 8 \mathrm{v}^{\mathrm{o}}$} & & Jehan Coedon & Alloué & 3 livres \\
\hline & & & Jehan Saffré & Procureur & 20 sous \\
\hline & & & Yvon Deraye & Receveur & 6 livres \\
\hline \multirow{4}{*}{$1 \mathrm{E} 674(5)$} & \multirow{4}{*}{$F^{\circ} 19 r^{\circ}$} & \multirow{4}{*}{1464} & Olivier Germand & Sénéchal & 10 livres \\
\hline & & & Gilles Lebel & Alloué & 5 livres \\
\hline & & & Fouques Coedon & Procureur & 5 livres \\
\hline & & & Yvon Deraye & Receveur & 10 livres \\
\hline \multirow{5}{*}{$1 \mathrm{E} 674(6)$} & \multirow{5}{*}{$\mathrm{F}^{\mathrm{o}} 30 \mathrm{v}^{\mathrm{o}}$} & \multirow{5}{*}{$\begin{array}{l}1462,1464 \text { et } \\
1465\end{array}$} & Pierres Le Boteiller & Sénéchal & 6 livres \\
\hline & & & Pierres Rembaut & Alloué & 3 livres \\
\hline & & & Jehan Saffray & Procureur & 3 livres \\
\hline & & & Jacquet Rouxel & Procureur & 3 livres \\
\hline & & & Jehan Paetral & Receveur & 6 livres \\
\hline \multirow{3}{*}{1 E $674(7)$} & $\mathrm{F}^{\circ} 47 \mathrm{r}^{\mathrm{o}}$ & 1470 & Pierre Rembaut & Alloué & 6 livres \\
\hline & \multirow[b]{2}{*}{$\mathrm{F}^{\mathrm{o}} 47 \mathrm{v}^{\mathrm{o}}$} & 1472 et 1473 & Pierres Le Bouteiller & Sénéchal & 6 livres \\
\hline & & $\begin{array}{l}1470,1471, \\
1472 \text { et } 1473\end{array}$ & Jehan Paetral & Receveur & 6 livres \\
\hline \multirow{3}{*}{1 E $674(8)$} & \multirow{3}{*}{$F^{\circ} 14 r^{o}$} & \multirow{3}{*}{1475} & Pierres Le Bouteiller & Sénéchal & 6 livres \\
\hline & & & Pierres Rembaut & Alloué & 3 livres \\
\hline & & & Jehan Paetral & Receveur & 6 livres \\
\hline $1 \mathrm{E} 674(9)$ & $\mathrm{F}^{\circ} 30 \mathrm{v}^{\mathrm{o}}$ & 1480 & Jehan Paetral & Receveur & 6 livres \\
\hline
\end{tabular}

a. "Nom du second des juges dans certaines juridictions. Le premier se nommait sénéchal et le troisième lieutenant. ", d'après le dictionnaire Atilf du CNRTL (Centre National de Ressources Textuelles et Lexicales). Disponible sur [http://www.cnrtl.fr/definition/allou\%C3\%A9] (consulté le 20 février 2019). 
mentionné dans les registres, en dehors des "nouvelles baillées ", ce qui appuie l'idée d'une origine extérieure à la châtellenie.

\section{Le patrimoine de la châtellenie de Saffré}

Comme les autres seigneuries de Bretagne méridionale, petites ou grandes, la châtellenie de Saffré constitue un ensemble dispersé, ici autour de trois noyaux principaux. Le premier reste sans conteste la paroisse de Saffré; 175 tenanciers y sont recensés dans le registre de 1456, répartis en trois sous-ensembles : 115 pour le fief de l'Hôpital, 21 pour le fief du Pont; les 39 derniers tenanciers sont présentés comme habitant Saffré, sans plus de précision quant au fief.

Toutes les nouvelles baillées se concentrent à Saffré. Dans le registre de 1456, 19 sont indiquées. La paroisse accueille également la métairie du Houssais ${ }^{29}$, implantée à $2 \mathrm{~km}$ au nord-est du bourg. Les " châtelains receveurs " s'installent donc, en toute logique, à Saffré pour administrer la châtellenie, comme l'indique le registre de $1476^{30}$.

Les données démographiques sont très difficiles à interpréter. Les éléments sont trop partiels ${ }^{31}$ et espacés dans le temps pour permettre d'envisager une étude précise. Les comptes ne servent du reste pas à recenser directement la population; ils gardent uniquement la trace des rentes et des droits pesant sur les biens concédés. Nous ne savons pas par conséquent combien de personnes exactement vivaient sur les exploitations - avec les enfants et les ascendants. Nous ne disposons pas non plus d'autres indications, comme pour le fouage ${ }^{32}$, qui permettraient d'avoir quelques ordres de grandeur. Rien n'exclut que les nouveaux preneurs aient auparavant exploité d'autres terres et qu'ils s'y maintiennent. Chaque nouvelle baillée leur permet d'étoffer leur patrimoine en lui adjoignant des parcelles contiguës ou en en cultivant d'autres. La perte des repères spatiaux, jamais clairement indiqués dans les comptes car par-

29. Le mot "métairie " est rarement employé dans les sources de la fin du Moyen Âge. Les actes bretons distinguent deux types d'exploitation suivant leur taille et leur implantation : les borderies d'un côté, les métairies de l'autre. Les borderies renvoient à des exploitations de taille réduite, implantées en marge des terroirs ou en situation périphérique, sur des espaces parfois difficiles à mettre en valeur. Les métairies désignent les grandes exploitations agricoles, regroupées autour d'un noyau proche le plus souvent du manoir seigneurial, et assurent un encellulement des hommes dans les pays de bocage (même si cet encellulement est loin d'être très contraignant). Sur ce sujet, nous renvoyons à : RАBOT, Brice, «Le développement des métairies dans le comté de Nantes à la fin du Moyen Âge (XIV'-Xve siècles) ", Annales de Bretagne et des Pays de l'Ouest, t. 124, $\mathrm{n}^{\mathrm{o}} 1$, mars 2017, p. 55-57.

30. Cf. supra note 17.

31. Les nouvelles baillées ne reprennent que le nom des preneurs; les listes de rentes ne précisent pas toujours les noms des assujettis.

32. Les comptes de la prévôté d'Ancenis fournissent des éléments précieux à ce sujet (Arch. dép. de Loire-Atlantique, E 261, E 262, E 263 et E 264; RABOT, Brice, Les structures seigneuriales..., op. cit., p. 296-297). 
faitement connus de tous ${ }^{33}$, ne permet pas de répondre à cette question. Une chose est en revanche plus assurée. Les " nouvelles baillées " sont attestées tout au long du $\mathrm{Xv}^{\mathrm{e}}$ siècle, ce qui souligne la lenteur et les hésitations de la reconstruction agraire après l'élan des années 1450 (tableau 4).

\section{Tableau 4 - « Nouvelles baillées » recensées dans les comptes}

\begin{tabular}{|c|c|c|c|}
\hline Cote & Référence dans le compte & Année & Nombre mentionné \\
\hline $1 \mathrm{E} 674(1)$ & $\mathrm{F}^{\circ} 2 \mathrm{r}^{\mathrm{o}}-4 \mathrm{v}^{\mathrm{o}}$ & 1446 & 12 \\
\hline $1 \mathrm{E} 674(2)$ & $\mathrm{F}^{\circ} 1 \mathrm{v}^{\mathrm{o}}-3 \mathrm{v}^{\mathrm{o}}$ & 1447 & 13 \\
\hline $1 \mathrm{E} 674(3)$ & $\mathrm{F}^{\circ} 8 \mathrm{r}^{\mathrm{o}}$ & 1456 & 19 \\
\hline $1 \mathrm{E} 674(4)$ & $\mathrm{F}^{\mathrm{o}} 1 \mathrm{v}^{\mathrm{o}}-2 \mathrm{v}^{\mathrm{o}}$ & 1457 & 12 \\
\hline $1 \mathrm{E} 674(6)$ & $\mathrm{F}^{\circ} 1 \mathrm{v}^{\mathrm{o}}-2 \mathrm{v}^{\mathrm{o}}$ & 1461 & 9 \\
\hline $1 \mathrm{E} 674(7)$ & $\mathrm{F}^{\circ} 2 \mathrm{v}^{\mathrm{o}}-4 \mathrm{r}^{\mathrm{o}}$ & 1470 & 11 \\
\hline $1 \mathrm{E} 674(8)$ & $\mathrm{F}^{\mathrm{o}} 2 \mathrm{r}^{\mathrm{o}}-3 \mathrm{v}^{\mathrm{o}}$ & 1475 & 12 \\
\hline
\end{tabular}

La paroisse de Sion-les-Mines, aux portes de la baronnie de Châteaubriant, contient le deuxième noyau du patrimoine foncier de la châtellenie de Saffré ${ }^{34}$. Les " châtelains receveurs " de Saffré ne présentent pas dans leurs comptes les levées de Sion-les-Mines. Sans doute limités ${ }^{35}$, les fiefs de cette paroisse n'en sont pas moins stratégiques. Situés sur la route reliant Châteaubriant au Grand-Fougeray, les domaines de Sion sont au carrefour du Castelbriantais, de l'est du comté de Nantes et du bas pays de la Vilaine. Les produits qui en sont issus peuvent aussi être exportés vers la France, toute proche. Les revenus étaient très certainement consignés dans des registres spécifiques, dont aucun ne nous est parvenu.

Les " châtelains receveurs " sont amenés à percevoir d'autres rentes à Nort-sur-Erdre ${ }^{36}$, à Joué-sur-Erdre ${ }^{37}$, à Avessac ${ }^{38}$ ou encore à Plessé ${ }^{39}$. L'assiette du prélèvement - jamais plus de 10 noms de tenancier(s) par paroisse - laisse penser à des fiefs secondaires, sans doute liés à des héritages ou à des afféagements très anciens. Les receveurs n'apportent là encore aucune précision sur l'étendue ou la nature des patrimoines.

33. Cette mémoire orale n'empêche pas certaines pertes : « [...] Et pour le fye Boussée, que l'on ne sceit où est et qui le tient, et doit par an XX [sic] au terme de Noël, qui valent pour IIII années VI soulz VIII deniers" (Arch. dép. de Loire-Atlantique, 1 E 674 (7), fo 46 vo).

34. Les receveurs citent toujours Sion-les-Mines après Saffré dans l'introduction des registres.

35. Aucun registre ne nous est parvenu pour la paroisse de Sion-les-Mines.

36. Il s'agit ici de droits de panage (Arch. dép. de Loire-Atlantique, 1 E 674 (4), fo $3 \mathrm{v}^{\circ}$ ).

37. Avec les panages dans la forêt du lieu.

38. Sur des tenures et des nouvelles baillées (Arch. dép. de Loire-Atlantique, 1 E 674 (5), fo $10 \mathrm{v}^{\mathrm{o}}$ ).

39. Des tenures sont associées au moulin de Plessé, avec en sus le paiement de dîmes en nature (Arch. dép. de Loire-Atlantique, 1 E 674 (6), fo $8 \mathrm{v}^{\circ}$ ). 
Quelques édifices seigneuriaux sont enfin recensés. Un moulin à drap est déclaré " ruineux ${ }^{40}$ " depuis de longues années à Plessé ${ }^{41}$. Des moulins à grains, un à eau et un à vent, sont mentionnés dans cette même paroisse ${ }^{42}$. Un autre moulin à vent est signalé à Saffré ${ }^{43}$. Les moulins ne sont pas exploités directement par les " châtelains receveurs ". Comme ailleurs en Bretagne méridionale, ils sont affermés, pour une durée de trois ans pour que soit réévalué régulièrement le montant des revenus perçus sous la forme d'enchères, mais aussi pour permettre aux preneurs de tirer profit de l'exploitation du moulin. L'enchère était en effet réglée dès l'octroi de la ferme sous la forme de setiers de seigle ${ }^{44}$, plus faciles à échanger sur les marchés extérieurs mais aussi beaucoup plus intéressants que les paiements en numéraire, fragilisés par l'érosion monétaire des années 1460-148045. La dispersion des moulins sur l'ensemble de la châtellenie justifie aussi cette préférence pour l'affermage, plus souple que la gestion directe. Le " châtelain receveur " peut ainsi se consacrer à d'autres tâches. Enfin, un four est attesté à Plessé, ce qui laisse voir l'importance de la paroisse dans la châtellenie, avec le regroupement de nombreux édifices seigneuriaux. Là encore, le four n'est pas exploité directement par le "châtelain receveur ", mais affermé.

Au total, le patrimoine de la châtellenie de Saffré apparaît très dispersé (figure 1), constitué d'une dizaine de fiefs qui vont de Saffré à Sion-lesMines, en passant par Plessé, Nort-sur-Erdre, Joué-sur-Erdre et Avessac ${ }^{46}$. Cette dissémination n'a pas d'incidence négative sur la gestion seigneuriale, car les " châtelains receveurs " savent s'entourer d'officiers compétents et faire appel, le cas échéant, à d'autres hommes ${ }^{47}$ pour tirer le plus de profit possible des terres et des biens concédés.

40. C'est-à-dire " en ruines ".

41. «[...] Et du moulin à draps dudit lieu, lesdits receveurs pour le temps de ce compte ne s'en chargent de nulle recepte, pour ce que ledit moulin est ruyneux long temps et. [...]" (Arch. dép. de Loire-Atlantique, 1 E 674 [5], fo 9 vo).

42. Id.

43. Arch. dép. de Loire-Atlantique, 1 E 674 (6), fo 8 ro.

44. RAвот, Brice, "Communautés rurales et édifices seigneuriaux en Bretagne méridionale aux XIV et XV ${ }^{\mathrm{e}}$ siècles ", Histoire et Sociétés Rurales, 50, 2 ${ }^{\mathrm{e}}$ semestre 2018, tableau 4, p. 90 .

45. Sur ce sujet, complexe, nous renvoyons aux présentations de : CinTRÉ, René, Les marches de Bretagne au Moyen Âge. Économie, guerre et société en pays de frontière (XIV $X V^{e}$ siècles), Pornichet, éd. Jean-Marie Pierre, 1992, p. 145-161; CoATIVy, Yves, La monnaie des ducs de Bretagne de l'an mil à 1499, Rennes, PUR, 2006, p. 370-372, 392-393; GALLET, Jean, La Seigneurie bretonne (1450-1680) : l'exemple du Vannetais, Paris, Publications de la Sorbonne, 1983, p. 286-290; LEGUAY, Jean-Pierre, MARTIN, Hervé, Fastes et malheurs..., op. cit., p. 210-227, 407-417; RABOT, Brice, " La gestion d'une seigneurie rurale en pays de Retz à la fin du Moyen Âge : la comptabilité de la Blanchardais ", Histoire et Sociétés Rurales, 45, juin 2016, p. 76-77; TOUCHARD, Henri, Le commerce maritime breton à la fin du Moyen Âge, Paris, Les Belles Lettres, 1967, p. 88-99.

46. Il ne faut pas oublier les rentes annuelles versées par le châtelain receveur de Saffré aux moines de La Meilleraye, entre Nort-sur-Erdre et Joué-sur-Erdre, au titre des " reconnaissances de fief".

47. C'est ce qu'indiquent les "mises et dépenses " avec les rétributions versées à des intermédiaires (officiers, tenanciers ou personnes extérieures comme les artisans) pour 
Figure 1 - Localisation des paroisses où sont mentionnés les fiefs et les autres biens concédés de la châtellenie de Saffré

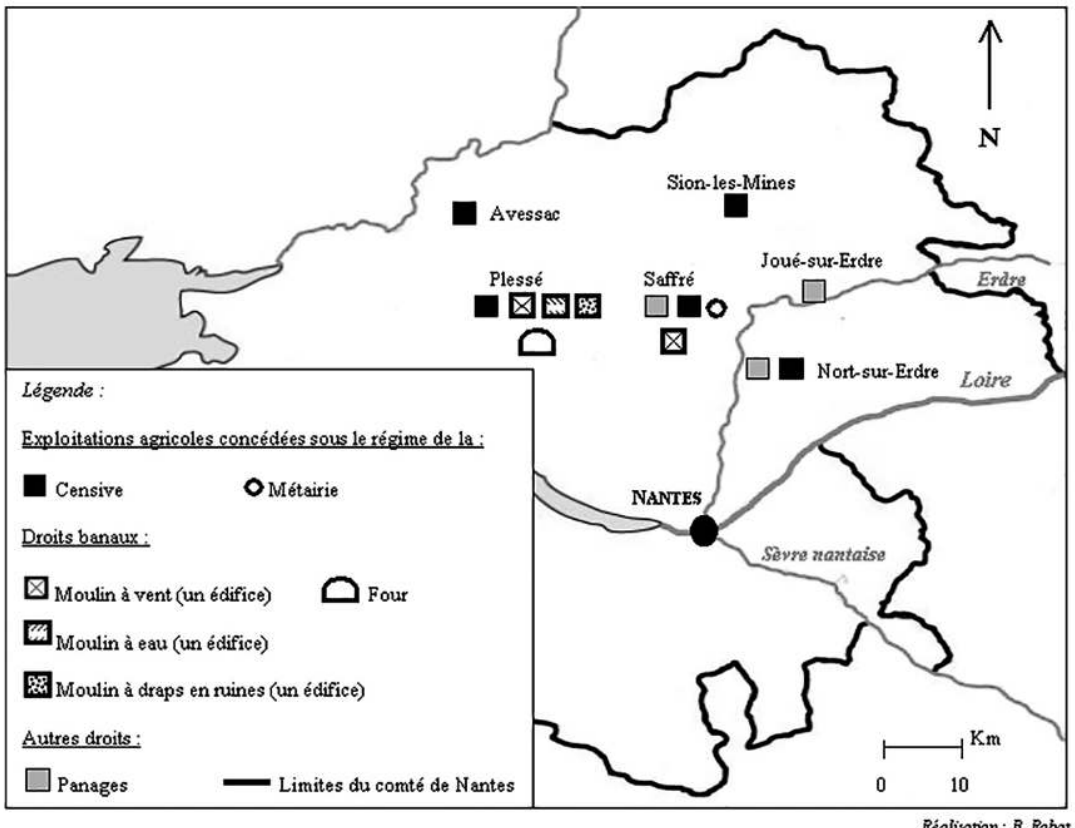

\section{Structures du prélèvement seigneurial}

Les comptes offrent un aperçu précis, pour les années 1460-1480, des structures du prélèvement en présentant les différents types de redevances, le calendrier des paiements ou encore les modalités de levée. Ces données peuvent être croisées avec celles des autres châtellenies de Bretagne méridionale.

\section{Principaux types de redevances}

Les livres de comptes de la châtellenie de Saffré sont semblables à ceux des autres seigneuries bretonnes. Les registres débutent toujours par la recension des redevances foncières. Les receveurs ne prennent pas soin de distinguer avec exactitude la nature des biens concédés. Comme ailleurs, il est très rare de voir apparaître le terme de " censive ". Les rédacteurs préfèrent utiliser les termes plus neutres de " masure ", de " maison avec herbergement ", " terres o appendances " pour désigner les biens concédés. Ces qualificatifs sont utilisés alternativement dans les longues listes des années 1440-1450, pour éviter leur répétition mais aussi pour mieux distin- 
guer les biens les uns des autres ${ }^{48}$. Les listes sont donc pour les receveurs des outils pratiques pour mettre en œuvre le prélèvement et pour veiller à la bonne perception de toutes les sommes dues. Ce trait, partagé avec les autres structures seigneuriales de Bretagne méridionale ${ }^{49}$, est révélateur des progrès accomplis en matière de gestion à la fin du $\mathrm{XV}^{\mathrm{e}}$ siècle, en particulier au tournant des années 1460-1470.

Figure 2 - Total des rentes " certaines » et « incertaines » connues

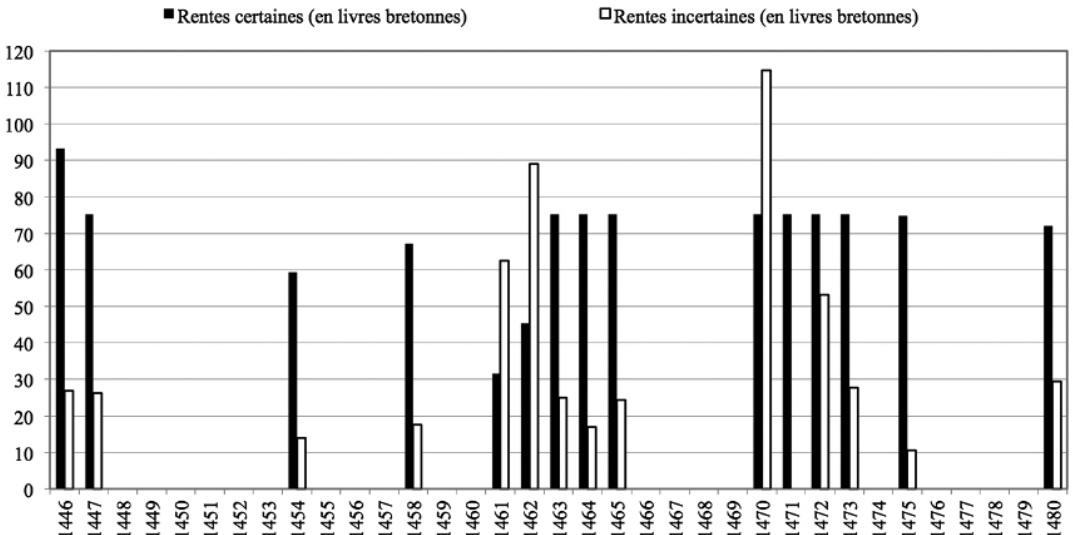

Les rentes foncières occupent une place majeure dans les recettes connues tout au long du $\mathrm{XV}^{\mathrm{e}}$ siècle. Les affrontements politiques et militaires des années 1460 d'une part, ceux des années 1480-1490 d'autre part, perturbent néanmoins les levées ${ }^{50}$. Les circuits économiques sont désorganisés; des marchands extérieurs prennent le relais des négociants bretons dans les échanges avec la France ${ }^{51}$, tandis que les mutations monétaires érodent peu à peu la valeur des rentes foncières ${ }^{52}$. La situation économique

48. Pour mieux distinguer les parcelles les unes des autres, les scribes retranscrivent les noms des tenanciers. Par exemple : Arch. dép. de Loire-Atlantique, 1 E 674 (1), fo 1 $\mathrm{v}^{\mathrm{0}}$ à $7 \mathrm{v}^{\mathrm{o}}$.

49. RABOT, Brice, Les structures seigneuriales..., op. cit., p. 310-311; Id., "Usages et enjeux des listes dans les seigneuries foncières de Bretagne méridionale à la fin du Moyen Âge (XIV $-\mathrm{XV}^{\mathrm{e}}$ siècles) ", Actes de la $4^{e}$ journée d'études de l'Association des Jeunes Chercheurs en Histoire (AJCH), Strasbourg, mai 2018, à paraître.

50. Sur les conséquences des crises de la seconde moitié du Xve siècle sur les revenus seigneuriaux, voir : RАВОт, Brice, Les structures seigneuriales..., op. cit., p. 259-285.

51. TouchaRD, Henri, Le commerce maritime breton..., op. cit., p. 257, 379-380.

52. RАВОт, Brice, Les structures seigneuriales..., op. cit., p. 277-280. Nous sommes beaucoup mieux renseignés pour la période postérieure à la série comptable de Saffré : les mentions de "foible monnoie " se multiplient dans les comptabilités seigneuriales à partir du milieu des années 1480. L'existence de gros de valeurs différentes, les références à la "foible monnoie " laissent penser que la Bretagne, comme d'autres provinces à l'instar du Toulousain, connaît des systèmes de monnaies de compte variés et simultanés, 
Figure 3 - Part des " rentes certaines » dans les recettes totales connues (en \%)

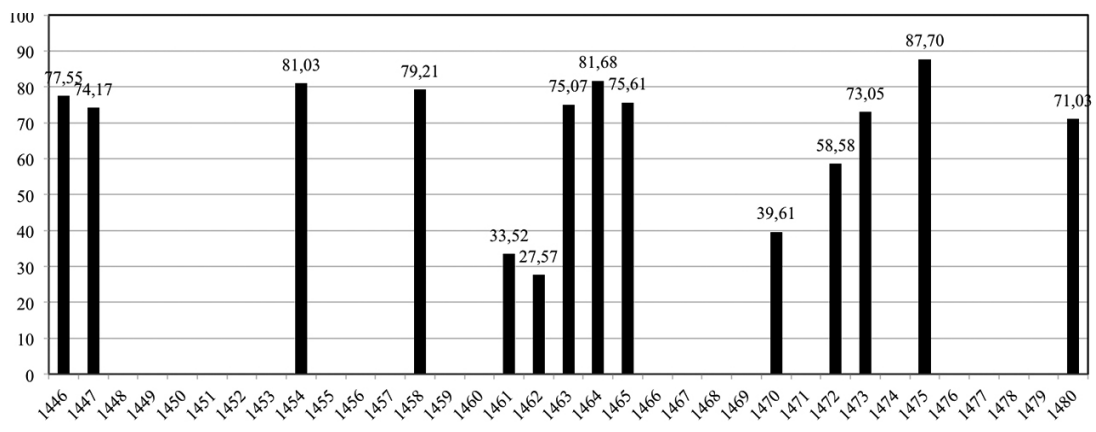

générale est donc très nettement dégradée à la fin $d u \mathrm{XV}^{\mathrm{e}}$ siècle. La part des rentes foncières dans les recettes totales en dehors des épisodes de crise (figures 2 et 3) est trompeuse. La figure 3 souligne un affaiblissement, modéré, dans les années 1470, avec moins de $75 \%$ de "rentes certaines " (ou rentes foncières) dans les recettes totales. La vente d'herbes, de bois, de denrées agricoles ou encore la mise aux enchères de certains prés explique ce léger repli des recettes certaines, compensées par les recettes incertaines, mises en œuvre au gré des besoins.

Les rentes incertaines occupent une place croissante dans les registres. La multiplication des enchères, des fermes ${ }^{53}$ et d'autres ventes exceptionnelles dans la seconde moitié du $\mathrm{XV}^{\mathrm{e}}$ siècle illustre les besoins sans cesse croissants des seigneurs et les difficultés pour les receveurs pour les financer. Ces derniers sont obligés d'user d'expédients, comme les ventes de bois, pour subvenir aux besoins sans remettre en cause le fragile équilibre des patrimoines (les biens concédés continuent de fournir une grande part des recettes connues).

Dans les années 1440, Yvon Deraye n'est pas contraint de vendre des bois pour financer les dépenses seigneuriales (tableau 5); il n'inscrit alors aucune ferme dans la partie " enchères ". Dans la seconde moitié $\mathrm{du} \mathrm{Xv}^{\mathrm{e}}$ siècle, les choses évoluent très rapidement. Yvon Deraye et Jehan Paetral multiplient les ventes de bois, en particulier les grands bois, c'està-dire les troncs pouvant servir à la construction et qui se vendent, pour cette raison, plus cher. Les recettes s'envolent par conséquent, pour

souvent délicats à distinguer (TOUCHARD, Henri, Le commerce maritime breton..., op. cit., p. 98-99; WolfF, Philippe, Commerces et marchands de Toulouse (vers 1350-vers 1450), Paris, 1954, p. 311; RABOT, Brice, Les structures seigneuriales..., op. cit., note 51, p. 277278). Ces systèmes utilisés en parallèle par les receveurs témoignent des incertitudes et des hésitations.

53. La Bretagne se caractérise, à la fin du Moyen Âge, par un " éclatement des modes de perception en une infinité de fermes " (LEGUAY, Jean-Pierre, MARTin, Hervé, Fastes et malheurs..., op. cit., p. 225). 
Tableau 5 - Recettes tirées des ventes de bois (mises aux enchères)

\begin{tabular}{|c|c|c|c|}
\hline Cote & $\begin{array}{l}\text { Référence dans le } \\
\text { compte }\end{array}$ & Année & $\begin{array}{c}\text { Montant perçu } \\
\text { (en livres bretonnes) }\end{array}$ \\
\hline 1 E 674 (1) & $\mathrm{F}^{\mathrm{o}} 5 \mathrm{v}^{\mathrm{o}}$ & 1446 & $4 \mathrm{l}$. \\
\hline $1 \mathrm{E} 674(2)$ & Fo $^{\circ} \mathrm{r}^{\mathrm{o}}$ & 1447 & $11.5 \mathrm{~s}$. \\
\hline 1 E 674 (3) & $\mathrm{F}^{\mathrm{o}} 9 \mathrm{r}^{\mathrm{o}}$ & 1456 & $\begin{array}{l}\text { "Item ne se charge } \\
\text { point ledit receveur } \\
\text { de nouvelles vantes } \\
\text { de boays pour ce } \\
\text { que n'en a este nulles } \\
\text { faictes depuix son } \\
\text { derroin compte." }\end{array}$ \\
\hline $1 \mathrm{E} 674$ (4) & $\mathrm{F}^{\mathrm{o}} 3 \mathrm{v}^{\mathrm{o}}$ & 1458 & $15 \mathrm{~s}$ \\
\hline 1 E 674 (5) & $\mathrm{F}^{\mathrm{o}} 3 \mathrm{r}^{\mathrm{o}}$ & 1462 & 96 l. 10 s. 10 d. \\
\hline 1 E 674 (5) & $\mathrm{F}^{\mathrm{o}} 3 \mathrm{v}^{\mathrm{o}}$ & 1463 & $108 \mathrm{l}$ \\
\hline 1 E $674(6)$ & Fo $^{\circ} 6 \mathrm{r}^{\mathrm{o}}$ & 1470 & $70 \mathrm{l}$ \\
\hline 1 E 674 (7) & Fo $^{\circ} 8 \mathrm{v}^{\mathrm{o}}-9 \mathrm{r}^{\mathrm{o}}$ & 1473 & 701. \\
\hline 1 E 674 (8) & $\mathrm{F}^{\mathrm{o}} 5 \mathrm{v}^{\mathrm{o}}$ & 1475 & 551. \\
\hline 1 E $674(9)$ & Fo $^{\circ} 15 \mathrm{v}^{\mathrm{o}}$ & 1480 & $22 \mathrm{l} .13 \mathrm{~s}$. \\
\hline
\end{tabular}

atteindre le pic de l'année 1463 avec un revenu de 108 livres. Les recettes de 1470 et 1475, qui atteignent plusieurs dizaines de livres elles aussi, sont sans commune mesure avec celles des précédentes décennies. Les besoins pressants, avec l'augmentation des dépenses, obligent à exploiter cette ressource rare et convoitée (les grands bois) :

" [...] [D'une autre main, dans la marge à gauche :] [Il en a perçu la vente] Et en ce que touche la vente des vieilx bieilz qui fut faicte à Jehan Gaudonner Michiel quatre solz, Yvon Hardi, pour la somme de L livres. Et de laquelle vente, cedit receveur, par sondit derroin, se procusa de s'en charger pour tant que les termes du poiement estoit a escheoir. Et, cy en droit, ne se charge de nouvelle recepte pour ce que Guillaume Desnoes, argentier et receveur general de mondit seigneur, en a fait le tout de ladicte recepte. Non obstant, il en est chargé, pour ce LV livres ${ }^{54}$."

Ce dernier exemple est révélateur : les sires de la Hunaudaye font face, en 1475, à une dépense exceptionnelle, à honorer immédiatement. Cela explique l'intervention du receveur principal, qualifié d'argentier et donc supérieur à Jehan Paetral. Une telle intervention reste toutefois inédite. Nous n'avons pas d'autre exemple dans la série comptable. La châtellenie de Saffré demeure une exception. Les autres séries disponibles pour le comté de Nantes ne relèvent pas d'intervention d'un officier supérieur se substituant au receveur. Les ventes restent toutefois limitées. Les receveurs ne parlent pas de futaies pour désigner les parcelles forestières, mais de

54. Arch. dép. de Loire-Atlantique, 1 E 674 (8), fo 5 vo. 
"bois et taillis ", autrement dit d'espaces en grande partie laissés à l'état sauvage et difficiles à exploiter. Sans doute aussi les bois n'étaient-ils pas de qualité suffisante pour alimenter de manière pérenne des circuits économiques. Il ne faut pas non plus négliger la concurrence des châtellenies et domaines proches, tels Le Gâvre ou Châteaubriant, très bien pourvus en espaces forestiers.

Les rentes "fossiles ${ }^{55}$ ", héritées de temps anciens, ne doivent pas être oubliées. Elles portent toutes des noms spécifiques qui permettent de bien les distinguer dans les comptes : il s'agit des " mangées ${ }^{56}$ ", des " donnes ${ }^{57}$ " ou encore des " chevroyes/chevroiz" ${ }^{58}$ ". Chacune est versée à un moment déterminé de l'année et repose sur un bien précis. Les receveurs ne s'attardent guère sur l'origine de ces rentes. La dernière mentionnée - les " chevroyes" - est peut-être un reste de corvées occasionnelles. Transformées en redevances annuelles, les " chevroyes " sont rattachées aux censives. Les " chevroyes" restent malgré tout très épisodiques. Les tenanciers doivent fournir à leurs frais des attelages, des charrettes et d'autres outils pour transporter les approvisionnements du manoir seigneurial. Les " chevroyes " s'avèrent contraignantes. Elles participent plus largement à la commercialisation des denrées issues du prélèvement avec les charrois. Les seigneurs tirent par conséquent un double profit avec la vente et l'absence de frais de transport.

Quelques journées de corvées sont enfin relevées. Deux journées par tenancier sont ainsi dues chaque année. Les " châtelains receveurs " de Saffré les remplacent volontiers par une somme en argent, plus facile à lever et à gérer ${ }^{59}$. Même modiques, ces redevances n'en demeurent pas moins essentielles. Elles rappellent à chaque assujetti l'autorité éminente, symbolique, des seigneurs. Ces derniers ne manquent jamais une occasion de le rappeler, car ils y prêtent une grande attention.

Les différentes redevances sont d'abord destinées à alimenter la grange et le grenier seigneurial. Elles permettent ensuite aux receveurs de s'acquitter des reconnaissances de fiefs, avec des rentes elles aussi diversifiées. Les " châtelains receveurs " de Saffré versent ainsi au châtelain de Fresnay,

55. Sur ce sujet et pour la Bretagne méridionale, voir : RABOT, Brice, Les structures seigneuriales..., op. cit., p. 149-150.

56. Arch. dép. de Loire-Atlantique, 1 E 674 (3), fo $2 \mathrm{v}^{\mathrm{o}}$.

57. Arch. dép. de Loire-Atlantique, 1 E 674 (3), fo $7 \mathrm{r}^{\circ}$.

58. Ces redevances sont mentionnées uniquement dans le registre rendu en 1476 : "Chevroiz Du devoir des chevroiz deuz chacun an à mondit seigneur en la paroesse de Saffré pour aller et affaires de mondit seigneur es paes de la rivière de Loire qui montent par an VIII charrettes, dont le mynu est décleré par le compte précédent, lequel devoir est deu au temps que mondit seigneur fait résidance en sa maison de Saffré et que les hommes subgiz à ce faire en sont suffisamment requis. Cedit receveur ne s'en charge de nulle pour ce que lesdiz subgiz n'ont point esté requis à ce faire. Et mesme que mondit seigneur n'y a fait aucune résidance durant le temps de cedit compte. [...] " (Arch. dép. de Loire-Atlantique, 1 E 674 (8), fo $7 \mathrm{v}^{\circ}$ ).

59. RАвот, Brice, Les structures seigneuriales..., op. cit., p. 144-146. 
en Plessé, des reconnaissances annuelles en numéraire à la place de rentes en nature :

«Item, compte ledit recepveurs [sic] avoir poié au chastelain de Fresnay, pour le rachat de ma feue damme, à qui Dieu pardoinne, pour les blez, faigns et pour les journées deues de rente pour marché avecques ledit chastelain à nous, en présence de monseigneur de la Guerche, la somme de vignt livres monnoie. Et, pour ce $[\ldots]^{60}$."

\section{Calendrier des paiements}

Le compte rendu en janvier 1456 (n. st.) offre une vision particulièrement précise des termes de paiement ${ }^{61}$. Nous avons, pour cette raison, choisi de prendre appui sur lui pour présenter le calendrier des levées (tableaux 6 et figure 4).

\section{Tableau 6 - Termes de paiement des " rentes certaines" selon le registre rendu en 1456 (n. st.)}

\begin{tabular}{|c|c|c|}
\hline \multirow{2}{*}{ Terme } & $\begin{array}{c}\text { Montant } \\
\text { (en livres bretonnes) }\end{array}$ & $\begin{array}{c}\text { Part parmi les recettes } \\
\text { totales en \% }\end{array}$ \\
\hline \multirow{2}{*}{ Chandeleur } & $15 \mathrm{~s}$. & 1,07 \\
\cline { 2 - 3 } & 2 boisseaux de pois & 100 \\
\hline Saint-Jean (24 juin) & $14 \mathrm{~s} .11 \mathrm{~d}$. & 1,07 \\
\hline Mi-août & $7 \mathrm{~s}$. & 0,50 \\
\hline $\begin{array}{c}\text { Décollation de saint Jean- } \\
\text { Baptiste (29 août) }\end{array}$ & $21.11 \mathrm{~s} .3 \mathrm{~d}$. & 3,66 \\
\hline \multirow{3}{*}{\begin{tabular}{c} 
Angevine (8 septembre) \\
\cline { 2 - 3 }
\end{tabular}} & $\begin{array}{c}29 \text { setiers } 9,5 \text { boisseaux de } \\
\text { grosse avoine }\end{array}$ & 100 \\
\cline { 2 - 3 } & 367 chapons & 100 \\
\cline { 2 - 3 } & 96 poules & 100 \\
\hline Toussaint & 10 l. $6 \mathrm{~s} .2 \mathrm{~d}$. & 14,72 \\
\hline Noël & $21 \mathrm{l} .3 \mathrm{~s}$. & 30,20 \\
\hline
\end{tabular}

Les " rentes certaines " en deniers s'organisent autour de deux termes principaux, la fin de l'été et Noël, en lien avec le calendrier agricole et liturgique. L'été et Noël sont deux moments forts dans la vie des communautés paysannes. Il est donc plus facile de procéder aux levées, notamment lorsque les rentes sont "à assembler ${ }^{62}$ ". Les " rentes certaines " en nature sont quant à elles levées au début du mois de septembre, soit

60. Arch. dép. de Loire-Atlantique, 1 E 674 (6), fo $20 \mathrm{r}^{\circ}$.

61. Arch. dép. de Loire-Atlantique, 1 E 674 (3), fo $1 r^{\circ}-8 r^{\circ}$.

62. RАВот, Brice, Les structures seigneuriales..., op. cit., p. 161-163. 
Figure 4 - Répartition (en \%) des termes de paiement des rentes certaines en deniers (paroisse de Saffré)

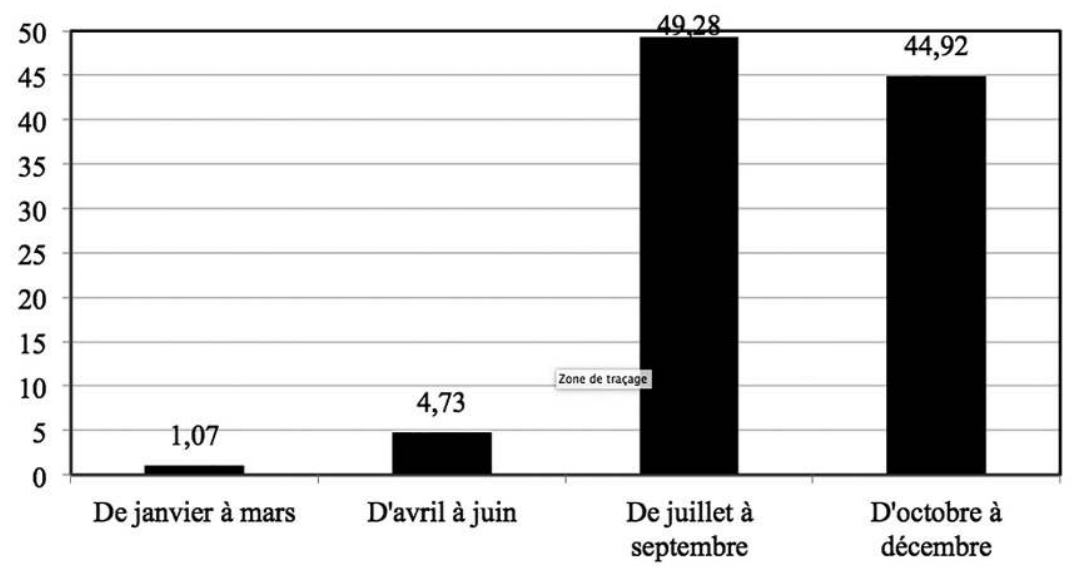

après les récoltes et avant que les paysans n'écoulent leurs productions sur les marchés locaux ou extérieurs. Tout est fait pour faciliter la perception mais aussi pour réduire le risque de fraude. Les volailles perçues en août alimentent les échanges avec l'extérieur. Ainsi organisées, les rentes permettent de disposer de revenus répartis tout au long de l'année pour faire face aux besoins, tout en dégageant quelques profits avec les ventes. Ce calendrier offre en outre une certaine souplesse pour les paysans. L'absence de levées au printemps facilite le passage de la "soudure ", toujours délicat, et limite le risque d'impayés ou d'arriérés. Les "nouvelles baillées ", effectuées au moment des successions ou des nouvelles concessions, ne modifient pas les termes ${ }^{63}$. Les deux plus communs restent fixés à l'Angevine et à Noël, sur le modèle des autres rentes. Les campagnes s'animent donc, périodiquement, de flux reliant les tenures à la résidence du receveur, où sont centralisés les prélèvements.

\section{Modalités de levée}

Les registres n'apportent que des informations indirectes. Contrairement aux aveux ou aux minus qui dénombrent les rentes et les droits, les comptes n'indiquent que les produits totaux perçus. Le découpage en rubriques, conjugué au faible nombre d'officiers, laisse penser que les rentes étaient portées au siège de la châtellenie, où officiait le receveur. Nous ne rencontrons pas dans les folios d'indications "par la main de", comme dans les aveux ou les dénombrements, qui laisseraient voir d'autres mécanismes de perception au sein des communautés paysannes. La mention de frérèches ou de tenanciers avec "leurs consors " ne permet pas

63. Arch. dép. de Loire-Atlantique, 1 E 674 (3), fo 8 ro. 
d'exclure les paiements par des intermédiaires ${ }^{64}$. Les rentes et les droits sont par conséquent payables sous la forme de rentes portables, comme ailleurs en Bretagne méridionale ${ }^{65}$.

La diversité des rentes et des prélèvements renforce cette idée. La multiplicité des dates de prélèvement, l'étendue du territoire de la châtellenie sont autant d'obstacles pour les levées directes. Rares sont les registres en Bretagne méridionale à indiquer clairement que les receveurs se rendaient dans les champs ou dans les exploitations pour percevoir les rentes foncières ${ }^{66}$. La position géographique de Saffré, au cœur de la châtellenie, facilite les charrois. En obligeant les tenanciers à se rendre à leurs frais en un point précis (cellier ou manoir seigneurial), les " châtelains receveurs " rappellent une nouvelle fois la position et l'autorité symbolique des sires de la Hunaudaye.

D'autres officiers sont mentionnés incidemment dans les comptes, tel Guillaume Desnoes ${ }^{67}$. Ils participent aux levées, en particulier lorsque les circonstances imposent de recourir à des expédients pour faire face à une dépense soudaine. Les comptes restent malheureusement trop discrets sur ces officiers. Aucun élément n'est indiqué sur l'origine, les revenus ou encore le territoire précis d'action de ces hommes au sein de la châtellenie.

\section{Évolutions de la gestion au $\mathrm{Xv}^{\mathrm{e}}$ siècle}

\section{Rentes seigneuriales et conjoncture}

Les remarques précédentes ont permis de rappeler les principaux cadres dans lesquels les " châtelains receveurs " de Saffré inscrivent leurs actions et leurs rapports de force. Il convient désormais de s'intéresser davantage au produit des rentes pour apprécier les impacts de la conjoncture. La continuité chronologique et la densité des informations permettent d'établir un bilan des recettes et dépenses connues ${ }^{68}$ (figure 5).

La figure 5 souligne la nette détérioration de la situation économique au tournant des années 1460. Les données sont trop espacées dans le temps pour que faire apparaître clairement des grandes tendances. Il faut donc comparer les données de la châtellenie de Saffré avec celles des autres séries, beaucoup plus étoffées chronologiquement, comme celles de la Blanchardais (figure 6). Les " châtelains receveurs " n'ont pas les outils

64. Les paiements par d'autres tenanciers donnent parfois lieu à quelques adaptations. Des réseaux de solidarité ou d'entraide peuvent être mis en œuvre, de manière ponctuelle, pour répartir au mieux les efforts du prélèvement. Seul compte le complet acquittement des redevances, ce qui explique que les aveux, les dénombrements ou les comptes restent très vagues sur les paiements "par la main de " (RABOT, Brice, Les structures seigneuriales..., op. cit., p. 161-163).

65. Ibidem, p. 153-178.

66. Ibid., p. 156-161.

67. Arch. dép. de Loire-Atlantique, 1 E 674 (8), fo 5 vº.

68. Les registres conservés n'éclairent que la situation avant et après les premières crises, celle de 1468 en particulier. 
Figure 5 - Solde des exercices comptables de la châtellenie de Saffré

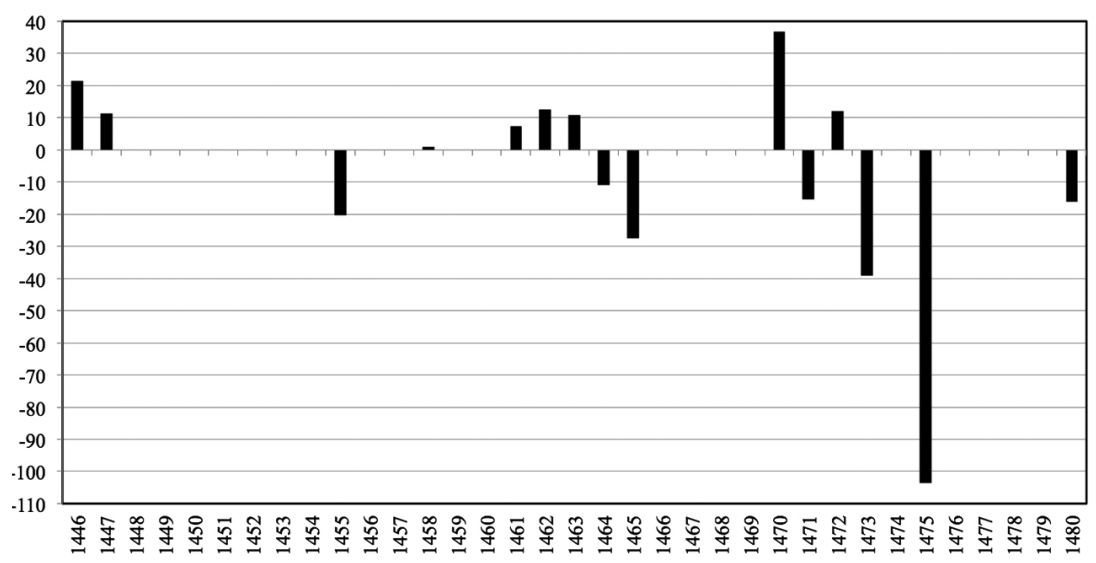

Figure 6 - Solde des exercices comptables de la Blanchardais

(en livres bretonnes) (Rabot, Brice, Les structures seigneuriales..., op. cit., graphique 34, p. 295 et le développement associé p. 294-295 [d'après Arch. dép. de Loire-Atlantique, 1 E 221, 1 E 222 et 1 E 223]).

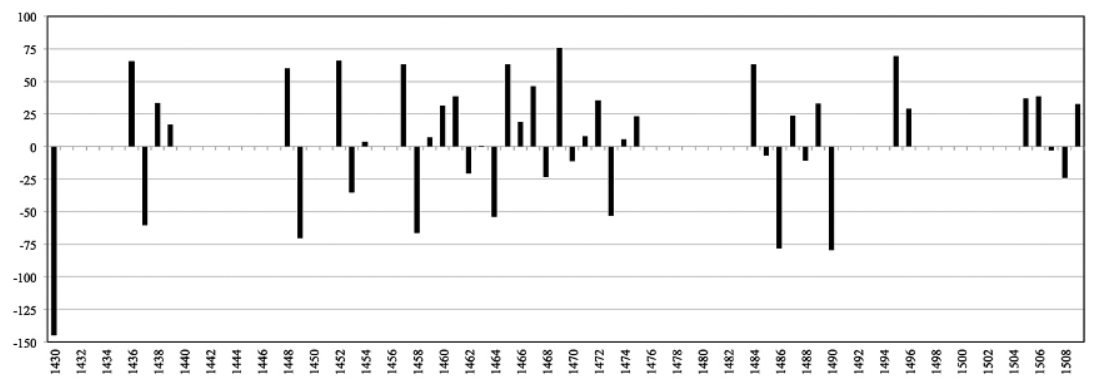

nécessaires pour anticiper les crises; ils ne peuvent donc pas s'y préparer. La part des "rentes certaines" dans le total des recettes connues bloque toute réorientation rapide des levées. Les tenanciers ne peuvent pas non plus payer de redevances supplémentaires, comme des surcens, car ils sont fragilisés par la désorganisation des circuits économiques et, surtout, par l'érosion monétaire comme nous l'avons déjà dit ${ }^{69}$. La gestion de la châ-

69. Le marc d'argent, sur lequel repose l'évaluation des monnaies de compte en livres bretonnes, est négocié en 1472 entre 8 livres 10 sous et 9 livres 10 sous. En 1479, il l'est entre 8 livres 10 sous et 10 livres 10 sous, avant de passer en mars 1487, pour les miseurs de la ville de Nantes, à 10 livres 10 sous (MORICE, Hyacinthe, Mémoires pour servir de preuves à l'histoire ecclésiastique et civile de Bretagne, Paris, rééd. 1974 (1 éd. 1742-1746), t. 3, p. 281; Arch. dép. de Loire-Atlantique, B 9, fo 208; B 10, fo 171 vo 215 ; Arch. municipales de Nantes, CC 101; cité par : TOUCHARD Henri, Le commerce maritime breton..., 
tellenie est aussi difficile car les "rentes incertaines " sont insuffisantes pour combler les pertes liées aux destructions ou aux fuites de paysans ${ }^{70}$. Les fermes de moulins, par exemple, sont payées en nature alors que l'entretien des édifices nécessite de lourds investissements en numéraire ${ }^{71}$. Pour obtenir ces liquidités, les receveurs devraient écouler les rentes en nature sur les marchés extérieurs; or ceux-ci sont soient bloqués, soient inaccessibles du fait des opérations militaires dans les années 1460 puis durant une grande partie des années 1480-1490. Les terres sont aussi plus irrégulièrement mises en valeur. Certaines sont abandonnées car pas assez productives, tandis que d'autres ne sont plus cultivées faute de bras du fait de la fuite des paysans ${ }^{72}$. La conjonction de ces différents éléments pèse sur les équilibres et accroît les déficits. Ces derniers ne doivent pour autant pas être exagérés. Comme le souligne l'examen de la comptabilité de la Blanchardais (figure 6), les déficits n'empêchent pas, une fois la crise passée, un sursaut avec un rétablissement des recettes.

L'examen des sommes perçues est tout aussi riche en enseignements. Les rentes foncières restent inchangées, du fait de la nature des concessions - sous le régime de la censive -, qui garantit en théorie de manière stable la jouissance de la terre et les biens aux preneurs tant qu'ils s'acquittent correctement et régulièrement des sommes dues. Les « châtelains receveurs " font donc appel à d'autres sources de revenus pour tenter de faire face aux crises, quitte à adapter les structures.

\section{Adaptations des structures}

Au tournant des années 1460, les fermes et les mises aux enchères se multiplient. Certaines demeurent à des montants identiques à ceux observés précédemment : les panages, par exemple, ne connaissent pas de brutales envolées au cours de la période (tableau 7), car ils sont liés au nombre de bêtes mises en pacage dans les espaces forestiers ou de prairies. L'insécurité ${ }^{73}$, la désorganisation des circuits économiques, l'absence de personnes pour encadrer ou assurer correctement les levées sont autant

op. cit., p. 295). Le gros d'argent ancien titre à 4 sous 6 deniers en mars 1490 et les gros neufs à 15 deniers. En février 1489, le gros d'argent tient encore à 2 sous 6 deniers. Voir : PocQueT Du Haut-Jussé Barthélemy, "Les emprunts de la Duchesse Anne à Julien Tierry (1489-1491) ", Annales de Bretagne, t. 69, 1962, p. 281; cité par Touchard Henri, Le commerce maritime breton..., op. cit., p. 296); Arch. dép. de Loire-Atlantique, 1 E 223 (5), fo 1 r $^{\circ}$.

70. RАВот, Brice, Les structures seigneuriales..., op. cit., p. 268-270.

71. RАвот, Brice, "Communautés rurales et édifices seigneuriaux... ", art. cité, p. 96-97.

72. Comme en 1468, lors de l'épisode du siège d'Ancenis et des opérations militaires qui se sont fait sentir jusque dans l'arrière-pays de Saffré (Arch. dép. de Loire-Atlantique, $1 \mathrm{E} 674$ [7], fo $\left.8 \mathrm{v}^{\circ}\right)$.

73. Comme au début des années 1470 dans les marches orientales : « [...] Et pour les dépans dudit chastelain qui fut par deux jours à choaisir ledit vin à Ancenis s'en retourner tant pour luy que son cheval avecques d'un homme quel mena quant et luy pour le bruit des gens d'armes que lors estoins ce païs [...] " (Arch. dép. de Loire-Atlantique, 1 E 674 [7], fo $28 r^{\circ}$ ). 
de freins qui expliquent la stagnation des cheptels ${ }^{74}$ dans les forêts de la châtellenie (tableau 8). Les montants sont aussi fixés par la coutume, ce qui empêche les receveurs d'appliquer des hausses brutales. Les panages entrent aussi en concurrence avec l'utilisation et la vente des " grands bois ", source fort lucrative, mais temporaire, de revenus dans ces temps troublés.

Tableau 7 - Montants perçus au titre du panage des « bêtes aumailles»

\begin{tabular}{|c|c|c|c|}
\hline Cote & $\begin{array}{l}\text { Référence } \\
\text { dans le } \\
\text { compte }\end{array}$ & Année & Montant perçu (en livres bretonnes) \\
\hline $1 \mathrm{E} 674(2)$ & $\mathrm{F}^{\circ} 4 \mathrm{v}^{\mathrm{o}}$ & 1447 & $7 \mathrm{l} .3 \mathrm{~s} .9 \mathrm{~d}$. \\
\hline $1 \mathrm{E} 674(3)$ & $\mathrm{F}^{\circ} 9 \mathrm{r}^{\mathrm{o}}$ & 1458 & $41.6 \mathrm{~s} .3 \mathrm{~d}$. \\
\hline $1 \mathrm{E} 674(5)$ & $\mathrm{F}^{\mathrm{o}} 4 \mathrm{v}^{\mathrm{o}}$ & 1463 & $23 \mathrm{l} .19 \mathrm{~s}$. \\
\hline $1 \mathrm{E} 674(7)$ & $\mathrm{F}^{\circ} 7 \mathrm{v}^{\mathrm{o}}$ & 1470 & $11.15 \mathrm{~s}$. \\
\hline 1 E $674(7)$ & $\mathrm{F}^{\mathrm{o}} 7 \mathrm{v}^{\mathrm{o}}$ & 1471 & $\begin{array}{l}\text { "Et pour le pasnaige desdits beufs de l'an mil } \\
\text { IIIICC LXXI n'en compte riens pour la guerre } \\
\text { qui en celuy temps estoit en ce païs tellement } \\
\text { que les abitans desdits pasnaiges et autres } \\
\text { estoins fuiz du païs pour ce que le seige } \\
\text { estoit lors a Encenis" }\end{array}$ \\
\hline $1 \mathrm{E} 674(7)$ & $\mathrm{F}^{\mathrm{o}} 7 \mathrm{v}^{\mathrm{o}}-8 \mathrm{r}^{\mathrm{o}}$ & 1472 & $11.5 \mathrm{~s}$ \\
\hline $1 \mathrm{E} 674(7)$ & $\mathrm{F}^{\circ} 7 \mathrm{v}^{\mathrm{o}}-8 \mathrm{r}^{\mathrm{o}}$ & 1473 & $1 \mathrm{l} .6 \mathrm{~s} .3 \mathrm{~d}$. \\
\hline 1 E $674(8)$ & $\mathrm{F}^{\circ} 5 \mathrm{r}^{\mathrm{o}}$ & 1475 & 18 s. 9 d. \\
\hline $1 \mathrm{E} 674(9)$ & $\mathrm{F}^{\circ} 15 \mathrm{r}^{\mathrm{o}}$ & 1480 & $3 \mathrm{l} .17 \mathrm{~s} .6 \mathrm{~d}$. \\
\hline
\end{tabular}

Tableau 8 - Nombre de bêtes mises en panage dans les bois de Saffré

\begin{tabular}{|c|c|c|c|c|c|}
\hline \multirow{2}{*}{ Cote } & \multirow{2}{*}{$\begin{array}{l}\text { Référence } \\
\text { dans le } \\
\text { compte }\end{array}$} & \multirow{2}{*}{ Année } & \multicolumn{3}{|c|}{ Montant perçu (en livres bretonnes) } \\
\hline & & & Bœufs & Juments & Porcs \\
\hline 1 E $674(3)$ & Fo $^{\circ} \mathrm{r}^{\mathrm{o}}$ & 1458 & 69 & & \\
\hline 1 E $674(6)$ & $\mathrm{F}^{\circ} 5 \mathrm{r}^{\mathrm{o}}$ & 1463 & & 19 & 460 \\
\hline 1 E $674(7)$ & $\mathrm{F}^{\mathrm{o}} 10 \mathrm{v}^{\mathrm{o}}$ & 1470 & 20 & & \\
\hline $1 \mathrm{E} 674(7)$ & $\mathrm{F}^{\mathrm{o}} 10 \mathrm{v}^{\mathrm{o}}$ & 1471 & 0 & & \\
\hline $1 \mathrm{E} 674(7)$ & $F^{\circ} 10 v^{o}$ & 1472 & 10 & & \\
\hline 1 E $674(7)$ & $\begin{array}{c}\mathrm{F}^{\circ} 10 \mathrm{v}^{0}- \\
11 \mathrm{r}^{\mathrm{o}}\end{array}$ & 1473 & 12 & & \\
\hline 1 E $674(8)$ & $\mathrm{F}^{\circ} 5 \mathrm{r}^{\mathrm{o}}$ & 1475 & 15 & & \\
\hline $1 \mathrm{E} 674(9)$ & $\mathrm{F}^{\circ} 15 \mathrm{r}^{\mathrm{o}}$ & 1480 & 62 & & \\
\hline
\end{tabular}

74. Les effectifs des porcs mis en panage ne sont pas tous connus du fait des enchères. Seul le montant total acquitté au départ est indiqué dans les registres. 
Les comptes de Saffré laissent voir des adaptations ponctuelles, dictées par les circonstances. Ces adaptations restent toutefois limitées. Elles se heurtent en premier lieu à l'inertie des structures; le maintien d'Yvon Deraye et de Jehan Paetral l'illustre parfaitement. Elles se heurtent plus largement aux embarras et aux hésitations des officiers et des autres agents chargés d'administrer les patrimoines seigneuriaux et de les faire fructifier. Pour opérer de tels ajustements, il faudrait une lisibilité à moyen terme et une stabilisation de la situation générale, comme en Anjou ou en Poitou dans la seconde moitié du XV $\mathrm{XV}^{\mathrm{e}}$ siècle avec la reconstruction agraire ${ }^{75}$. C'est tout le contraire qui se produit ici ${ }^{76}$.

\section{Conséquences des crises sur le prélèvement}

La première conséquence, la plus grave aussi, est l'affaissement des recettes. La valeur réelle du denier ne cesse de s'affaiblir à partir des années 1470. Les crises monétaires, les choix des ducs bretons pour financer la guerre et leur politique de rivalité avec la France pèsent de plus en plus lourd sur l'économie. L'essentiel des érosions monétaires se produit dans les années 1480-1490, autrement dit pour des années postérieures à la série conservée pour Saffré. Les premiers signes de ces changements brutaux peuvent être décelés à la lecture des graphiques précédents (figures 2, 3 et 5). La montée des "rentes incertaines " dans les recettes connues, les contestations de rentes avec les demandes de décharge, formulées plus régulièrement par les receveurs dans les années 1470 (figure 7$)^{77}$, sont des traces indirectes de ces nécessaires adaptations.

Les indications annexes portées dans les registres sont aussi une source d'information. Les annotations des auditeurs, portées en marge avec une couleur différente pour bien les distinguer de celles du receveur, montrent l'examen de plus en plus scrupuleux des registres, à mesure que la conjoncture se tend. Les demandes de décharge ou " rabat " sont plus précises et davantage motivées par les " châtelains receveurs " pour limiter au maximum les risques de rejet par les auditeurs ${ }^{78}$. Les inquiétudes grandissent,

75. Le Mené, Michel, Les campagnes angevines à la fin du Moyen Âge (1350-1530). Étude économique, Nantes, Cid éditions, 1982, p. 188-189, 213, 220-221; MERLE, Louis, La métairie et l'évolution agraire de la Gâtine poitevine de la fin du Moyen Âge à la Révolution, Paris, sevpen, 1958, p. 96.

76. RАВОт, Brice, Les structures seigneuriales..., op. cit., p. 259-285; Id., "Crises et recompositions des campagnes de Bretagne méridionale à la fin du Moyen Âge ", dans Fr. LAGET, Ph. Josserand et B. RABOT (dir.), Entre horizons terrestres et marins. Sociétés, campagnes et littoraux de l'Ouest atlantique. Mélanges offerts à Jean-Luc Sarrazin, Rennes, PUR, 2017, p. 143-152.

77. Par exemple : « [...] Item, pour la meson Olivier de la Morteraye que tient à présent Yvon Deraye et doibt selon ledit rantier VIII soulz et celui Deraye dit n'en devoir que sept soulz, en demande rabat ledit repceveur XII deniers [...] " (Arch. dép. de Loire-Atlantique, 1 E $\left.674(3), \mathrm{f}^{\circ} 17 \mathrm{r}^{\circ}\right)$.

78. Pour ne citer qu'un exemple : «[...] Item, demande ledit repceveur lui estre rabatu et mis en descharge et rabat neuff sols de rente dont c'est trouvé chargé en ces comptes 
Figure 7 - Montants des "rabats » et décharges demandés par les receveurs (Arch. dép. de Loire-Atlantique, 1 E 674 [1], $f^{o} 7 r^{o}$ )

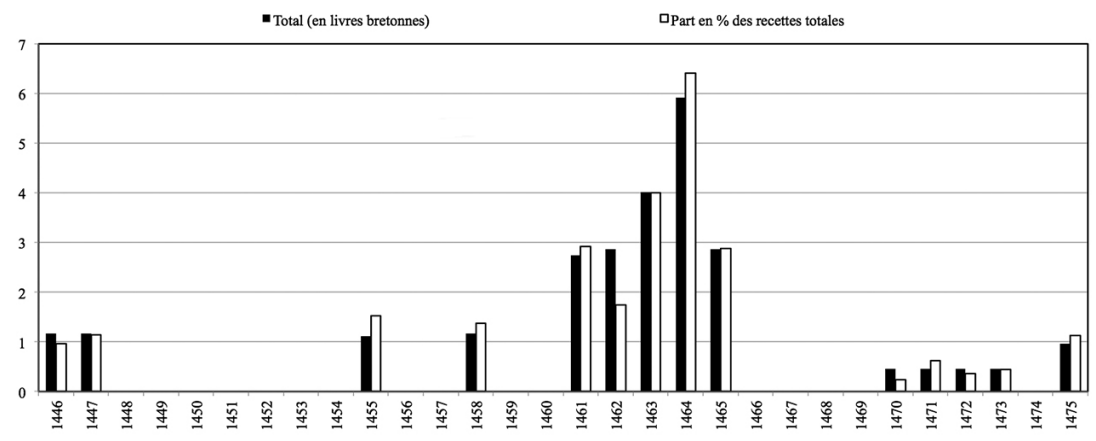

Les recettes de l'année ont été perçues directement par le grénetier. Elles n'apparaissent donc pas dans le compte de l'année 1446-1447.

tandis que les gages des officiers seigneuriaux ne progressent pas, voire diminuent pour le sénéchal ou le receveur (tableau 3). On comprend dans ces conditions que Jehan Paetral cherche l'accord des auditeurs pour ne pas avoir à avancer les sommes non perçues. Sans être exagérées, les tensions de la seconde moitié du $\mathrm{XV}^{\mathrm{e}}$ siècle pèsent beaucoup plus sur les budgets et sur les mentalités. Les " nouvelles baillées " compensent en partie les pertes, car elles permettent de récupérer une partie des sommes dues par les tenanciers. Elles ne couvrent toutefois pas toutes les pertes.

Comme nous l'avons dit, les crises ne se traduisent pas par une hausse brutale des prélèvements en numéraire. L'examen des revenus tirés de la métairie du Houssais éclaire d'autres aspects. Les redevances perçues sont constituées de volailles (poules et chapons) et, surtout, de céréales (froment, seigle et avoine). Ces redevances alimentent d'abord la table seigneuriale; les surplus sont ensuite écoulés sur les marchés extérieurs (tableaux 9, 10,11, 12 et 13) pour obtenir d'autres ressources.

précédents et dempuix qui fut institué et mis oudit office (quelx) quelx sont deuz comme leurdit prix cause et tenement de Beaumaunes et en la moitié du fait combien qu'il s'en sceit chargé de xxv soulz. Il n'en est deu que deiz et ouict souls plus n'en a receu et receveurs ne aultres de par avant lui ainxin est qu'est error et charge ad ce recepveurs. Suppliant mondit seigneur et les audicteurs de ses \{présentes\} visites et gardes es rolles et comptes précédens et sur ce pourvoays et faire la provision que en tel cas appartient par ant nostre sire qu'il doint à mondit seigneur, à ma dame, sa compaigne, et à toutes leurs [...] noble lingnée, bonne vie et longue. Et à messeigneurs des comptes, prospérité et senté. [Dans la marge, à gauche, d'une autre main :] Refuse, pour ce que les précédens n'en ont point eu de rabat " (Arch. dép. de Loire-Atlantique, 1 E 674 (6), fo 29 ro). En 1474, Jehan Paetral rappelle aussi que 24 livres de " rabat " ont été refusées par les auditeurs pour des frais de justice (Arch. dép. de Loire-Atlantique, 1 E 674 (7), fo 49 ro). 
Tableau 9 - Recettes en froment tirées de la métairie du Houssais ${ }^{79}$ (Arch. dép. de Loire-Atlantique, 1 E 674 [1], fo $7 r^{o}$ )

\begin{tabular}{|c|c|c|c|}
\hline Cote & Référence dans le compte & Année & Boisseaux perçus \\
\hline $1 \mathrm{E} 674(2)$ & Fo $^{\circ} \mathrm{r}^{\circ}$ & 1447 & 100,75 \\
\hline $1 \mathrm{E} 674(6)$ & $\mathrm{F}^{\circ} 7 \mathrm{v}^{\mathrm{o}}$ & 1461 & 20 \\
\hline $1 \mathrm{E} 674(6)$ & $F^{\circ} 7 v^{o}$ & 1463 & 5,5 \\
\hline 1 E $674(6)$ & $F^{\circ} 7 v^{o}$ & 1464 & 10 \\
\hline 1 E $674(6)$ & $\mathrm{F}^{\circ} 7 \mathrm{v}^{\mathrm{o}}$ & 1465 & 74 \\
\hline $1 \mathrm{E} 674(7)$ & $\mathrm{F}^{\circ} 7 \mathrm{r}^{\mathrm{o}}$ & 1471 & 3 \\
\hline 1 E $674(7)$ & $F^{\circ} 7 r^{\circ}$ & 1472 & 21 \\
\hline $1 \mathrm{E} 674(7)$ & $\mathrm{F}^{\circ} 7 \mathrm{r}^{\mathrm{o}}$ & 1473 & 7 \\
\hline $1 \mathrm{E} 674(7)$ & $F^{\circ} 7 r^{\circ}$ & 1474 & 7 \\
\hline $1 \mathrm{E} 674(7)$ & $\mathrm{F}^{\circ} 7 \mathrm{r}^{\circ}$ & 1475 & 27,5 \\
\hline 1 E $674(9)$ & $\mathrm{F}^{\circ} 20 \mathrm{r}^{\circ}$ & 1480 & 27,5 \\
\hline
\end{tabular}

Les recettes de l'année ont été perçues directement par le grénetier. Elles n'apparaissent donc pas dans le compte de l'année 1446-1447.

Tableau 10 - Recettes en seigle tirées de la métairie du Houssais

\begin{tabular}{|c|c|c|c|}
\hline Cote & Référence dans le compte & Année & Boisseaux perçus \\
\hline 1 E 674 (2) & $\mathrm{F}^{\circ} 5 \mathrm{r}^{\mathrm{o}}$ & 1447 & 132 \\
\hline $1 \mathrm{E} 674(6)$ & $\mathrm{F}^{\circ} 7 \mathrm{v}^{\mathrm{o}}$ & 1461 & 120 \\
\hline 1 E $674(6)$ & $\mathrm{F}^{\mathrm{o}} 7 \mathrm{v}^{\mathrm{o}}$ & 1462 & 36 \\
\hline $1 \mathrm{E} 674(6)$ & $\mathrm{F}^{\circ} 7 \mathrm{v}^{\mathrm{o}}$ & 1463 & 237 \\
\hline $1 \mathrm{E} 674(6)$ & $\mathrm{F}^{\circ} 7 \mathrm{v}^{\mathrm{o}}$ & 1464 & 82 \\
\hline $1 \mathrm{E} 674(6)$ & $\mathrm{F}^{\circ} 7 \mathrm{v}^{\mathrm{o}}$ & 1465 & 143 \\
\hline $1 \mathrm{E} 674(7)$ & $\mathrm{F}^{\mathrm{o}} 7 \mathrm{v}^{\mathrm{o}}$ & 1470 & 89 \\
\hline 1 E 674 (7) & $\mathrm{F}^{\mathrm{o}} 7 \mathrm{v}^{\mathrm{o}}$ & 1471 & 53 \\
\hline 1 E $674(7)$ & $\mathrm{F}^{\mathrm{o}} 7 \mathrm{v}^{\mathrm{o}}$ & 1472 & 52 \\
\hline $1 \mathrm{E} 674(7)$ & $\mathrm{F}^{\circ} 7 \mathrm{v}^{\mathrm{o}}$ & 1473 & 57 \\
\hline 1 E $674(7)$ & $\mathrm{F}^{\circ} 7 \mathrm{v}^{\mathrm{o}}$ & 1474 & 33 \\
\hline 1 E $674(8)$ & $\mathrm{F}^{\mathrm{o}} 7 \mathrm{v}^{\mathrm{o}}$ & 1475 & 55 \\
\hline 1 E $674(9)$ & $\mathrm{F}^{\mathrm{o}} 20 \mathrm{r}^{\mathrm{o}}$ & 1480 & 55 \\
\hline
\end{tabular}

79. Les recettes de l'année ont été perçues directement par le grénetier. Elles n'apparaissent donc pas dans le compte de l'année 1446-1447 (Arch. dép. de Loire-Atlantique, 1 E $674(1)$, fo $\left.7 \mathrm{r}^{\circ}\right)$. 
Tableau 11 - Recettes en avoine tirées de la métairie du Houssais

\begin{tabular}{|c|c|c|c|c|}
\hline \multirow{2}{*}{ Cote } & $\begin{array}{c}\text { Référence dans } \\
\text { le compte }\end{array}$ & \multirow{2}{*}{ Année } & \multicolumn{2}{|c|}{ Boisseaux perçus } \\
\cline { 4 - 5 } & Grosse avoine & Avoine menue \\
\hline $1 \mathrm{E} 674(2)$ & $\mathrm{F}^{\circ} 5 \mathrm{v}^{\mathrm{o}}-6 \mathrm{r}^{\circ}$ & 1447 & 39 & 41 \\
\hline $1 \mathrm{E} 674(3)$ & $\mathrm{F}^{\circ} 11 \mathrm{r}^{\mathrm{o}}$ & 1454 & 30 & \\
\hline $1 \mathrm{E} 674(4)$ & $\mathrm{F}^{\circ} 4 \mathrm{v}^{\mathrm{o}}$ & 1465 & 22 & \\
\hline $1 \mathrm{E} 674(7)$ & $\mathrm{F}^{\circ} 10 \mathrm{r}^{\mathrm{o}}$ & 1470 & 16 & \\
\hline $1 \mathrm{E} 674(7)$ & $\mathrm{F}^{\circ} 9 \mathrm{r}^{\circ}$ & 1471 & 26 & \\
\hline $1 \mathrm{E} 674(7)$ & $\mathrm{F}^{\circ} 9 \mathrm{r}^{\circ}$ & 1473 & 7 & \\
\hline $1 \mathrm{E} 674(7)$ & $\mathrm{F}^{\circ} 9 \mathrm{r}^{\circ}$ & 1474 & 9 & \\
\hline $1 \mathrm{E} 674(7)$ & $\mathrm{F}^{\circ} 9 \mathrm{r}^{\circ}$ & 1475 & 27,5 & \\
\hline $1 \mathrm{E} 674(9)$ & $\mathrm{F}^{\circ} 20 \mathrm{r}^{\circ}$ & 1480 & 27,5 & \\
\hline
\end{tabular}

Tableau 12 - Recettes en volailles tirées de la métairie du Houssais

\begin{tabular}{|c|c|c|c|c|}
\hline Cote & $\begin{array}{c}\text { Référence dans } \\
\text { le compte }\end{array}$ & Année & Poule & Chapons \\
\hline $1 \mathrm{E} 674(1)$ & $\mathrm{F}^{\circ} 8 \mathrm{v}^{\mathrm{o}}$ & 1446 & 1 & \\
\hline $1 \mathrm{E} 674(1)$ & $\mathrm{F}^{\circ} 8 \mathrm{v}^{\mathrm{o}}$ & 1447 & 1 & \\
\hline $1 \mathrm{E} 674(3)$ & $\mathrm{F}^{\circ} 14 \mathrm{r}^{\mathrm{o}}$ & 1454 & 1 & \\
\hline $1 \mathrm{E} 674(4)$ & $\mathrm{F}^{\circ} 5 \mathrm{v}^{\mathrm{o}}$ & 1465 & 1 & \\
\hline $1 \mathrm{E} 674(7)$ & $\mathrm{F}^{\circ} 10 \mathrm{r}^{\circ}$ & 1472 & 1 & 2 \\
\hline $1 \mathrm{E} 674(7)$ & $\mathrm{F}^{\circ} 10 \mathrm{r}^{\circ}$ & 1473 & 1 & 2 \\
\hline $1 \mathrm{E} 674(7)$ & $\mathrm{F}^{\circ} 10 \mathrm{r}^{\circ}$ & 1474 & 1 & 2 \\
\hline $1 \mathrm{E} 674(8)$ & $\mathrm{F}^{\circ} 25 \mathrm{r}^{\circ}$ & 1475 & 1 & \\
\hline
\end{tabular}

Tableau 13 - Autres recettes en nature tirées de la métairie du Houssais (en boisseaux)

\begin{tabular}{|c|c|c|c|c|}
\hline Cote & Référence dans le compte & Année & Orge & Fèves \\
\hline $1 \mathrm{E} 674(6)$ & $\mathrm{F}^{\circ} 8 \mathrm{v}^{\mathrm{o}}$ & 1470 & 11 & \\
\hline $1 \mathrm{E} 674(7)$ & $\mathrm{F}^{\circ} 17 \mathrm{v}^{\mathrm{o}}$ & 1471 & & 2 \\
\hline $1 \mathrm{E} 674(7)$ & $\mathrm{F}^{\circ} 18 \mathrm{r}^{\mathrm{o}}$ & 1473 & 5 & 7 \\
\hline
\end{tabular}

Les receveurs privilégient donc, à partir des années 1460, les recettes en nature, plus stables ${ }^{80}$ et pouvant être vendues à différents moments à l'extérieur. Elles offrent en outre la possibilité de profiter de la hausse des

80. Les rentes en nature sont plus stables que celles en numéraire, dans le contexte de l'érosion monétaire, car les sommes demandées aux tenanciers étaient fixes semblet-il. Aucune redevance à part de fruits n'est en effet indiquée dans la série comptable de Saffré. Cela ne veut pas dire, pour autant, que toutes les récoltes étaient bonnes chaque année. 
prix, comme l'on peut le constater avec la vente des produits de la métairie $^{81}$. On voit également les cultures se diversifier, avec le développement de l'orge et des fèves. Les prélèvements sur ces cultures apparaissent dans la série comptable en 1468, soit après la première grave crise militaire avec la France (invasion des marches orientales du comté de Nantes et siège d'Ancenis). Les nouvelles denrées sont aussi exigées au moment où des paysans, réfugiés des "marches " ${ }^{82}$, s'installent dans l'arrière-pays nantais. Dès 1475 , les rentes en orge ou en fèves sont abandonnées au profit des céréales, plus lucratives pour les seigneurs. Enfin, en 1475, deux chapons apparaissent dans les comptes aux côtés de la poule annuelle. Les adaptations ne concernent pas uniquement les prélèvements, elles touchent aussi aux conditions d'exploitation pour permettre les ajustements au gré des besoins et des circonstances. La métairie est ainsi affermée pour 6 ans à partir de cette même date ${ }^{83}$. Tous ces éléments soulignent la gestion pragmatique de la métairie, qui guide l'action de Jehan Paetral. Ce trait se retrouve là encore pour les autres châtellenies de Bretagne méridionale ${ }^{84}$.

Les crises fragilisent enfin les situations paysannes. Certains, néanmoins très rares, n'ont aucun moyen pour faire face à leurs obligations :

"[...] Item, que je me soyes chargé en ma charge pour ung nommé Symon Guillet, qui demeure en l'oustel de ma dicte dame au Houssay, ge m'en descharge pour ce que il n'a rien et n'a de quoy poyer.

[D'une autre main, en dessous :] Item, demande luy estre rabatu LII soulz VI deniers dont fut chargé à son derrain compte de la parcelle Baillet, donnée à Guillaume de Lalende, pour ce que dit n'en avoir receu que VI livres et qu'il y a esté deffendu de ma dicte dame comme il dit de n'en plus en lever et pour ce LII soulz VI deniers.

[Dans la marge, à gauche, de la même main :] Déport, en attendant savoir si ledit de Lalende de a esté deffendu de [...] comme dit est ${ }^{85}$."

On retrouve, dans ce dernier exemple, l'examen de plus en plus méticuleux des auditeurs, qui reportent leur décision de décharge pour vérifier les éléments sur le terrain. La nouvelle attention portée au contrôle n'est pas nécessairement négative. Elle pose les bases de la reconstruction des structures d'autorité après les crises de la fin du Xve siècle, en mettant en œuvre et en promouvant des outils plus stricts et plus sûrs pour exiger le paiement de toutes les rentes et de tous les droits dus. Ces évolutions prennent du temps. Il faut attendre la première moitié du $\mathrm{XVI}^{\mathrm{e}}$ siècle pour voir s'épanouir les premiers effets de cette reprise en main des structures

81. Nous n'avons plus d'indications de rentes en deniers après 1458 : 5 l. 8 s. 7 d. sont perçus en 1446 et 1447, 5 l. 15 s. 7 d. en 1458 en sus des prélèvements en nature recensés dans les graphiques 11 à 15 .

82. Arch. dép. de Loire-Atlantique, 1 E 674 (7), fo 8 vo -9 ro.

83. Nous ne savons pas, avant les années 1470, quelle était la durée de concession de la métairie de la Houssais.

84. RАвОт, Brice, Les structures seigneuriales..., op. cit., p. 82-91, 275-285.

85. Arch. dép. de Loire-Atlantique, 1 E 674 (3), fo 17 vo. 
de gestion. Les seigneurs exigent ensuite des prélèvements plus lourds et exercent une autorité plus étroite sur leurs tenanciers ${ }^{86}$.

Les comptes de la châtellenie de Saffré sont un exemple unique de stabilité dans la gestion en Bretagne méridionale. Deux hommes seulement se chargent d'établir et de rendre, de manière quasi-continue, les registres de compte sur les dix-huit années connues. Cette stabilité est à la fois une preuve de confiance mais aussi un outil majeur pour mettre en valeur un patrimoine peu diversifié et très dispersé. Les rentes foncières constituent près de la moitié des recettes connues, ce qui place la châtellenie de Saffré au-dessus de la moyenne des structures similaires du comté de Nantes $\mathrm{au} \mathrm{XV}^{\mathrm{e}}$ siècle. L'examen des comptes de Saffré atteste également d'un lent mouvement de reconstruction agraire dans la première moitié du Xve siècle, qui se poursuit jusqu'au tournant des années 1460. Les premières crises mettent à mal ces entreprises en érodant les rentes foncières et en fragilisant les modes de gestion avec l'accroissement des déficits dans les années 1470 , à la veille de la plus grave crise du $\mathrm{Xv}^{\mathrm{e}}$ siècle avec la guerre de 14871491. Les receveurs tentent d'y faire face en élargissant et en réorientant les sources de prélèvement, avec la mise en place de nouvelles rentes (comme les rentes en orge ou en fèves) ou en usant de pragmatisme (" nouvelles baillées " qui permettent de compenser une partie des pertes en réattribuant les tenures laissées ou tombées vacantes). Au début des années 1480, quand se clôt la série comptable, la châtellenie apparaît déjà fragilisée alors que les crises les plus dures ne se sont pas encore manifestées.

\section{RÉSUMÉ}

Les progrès accomplis en matière de comptabilité tout au long du $\mathrm{xv}^{\mathrm{e}}$ siècle permettent de porter de nouveaux regards sur l'économie seigneuriale. La châtellenie de Saffré, qui n'a pas fait jusqu'alors l'objet de travaux universitaires, est tout à fait emblématique. Les comptes des receveurs éclairent les crises des années 1460-1470, ainsi que les modalités de mise en valeur agricole, avec l'importance des fermes, des enchères et des métairies. Il est ainsi possible de mettre en évidence les singularités et les points communs en comparant Saffré aux autres châtellenies.

\section{ABSTRACT}

The progress made in accounting throughout the fifteenth century made it possible to take a new look at the seigneurial economy. The castle of Saffré, which has not been the subject of academic work until now, is quite emblematic. The receivers' accounts shed light on the crises of the years 1460-1470, as well as on the modalities of agricultural development, highlighting the importance of farms, auctions and tenants. It is thus possible to highlight the singularities and common points by comparing Saffré with the other similar examples.

86. GaLlet, Jean, Seigneurs et paysans bretons du Moyen Âge à la Révolution, Rennes, Éditions Ouest-France Université, 1992, p. 314-316. 